\title{
Article
}

\section{Superficial Characteristics and Functionalization Effectiveness of Non-Toxic Glutathione-Capped Magnetic, Fluorescent, Metallic and Hybrid Nanoparticles for Biomedical Applications}

\author{
C. Fernández-Ponce ${ }^{1,+}{ }^{\circledR}$, J. M. Mánuel ${ }^{2,+}{ }^{\dagger}$, R. Fernández-Cisnal ${ }^{1}$, E. Félix ${ }^{2}$, J. Beato-López ${ }^{3}{ }^{(D}$,

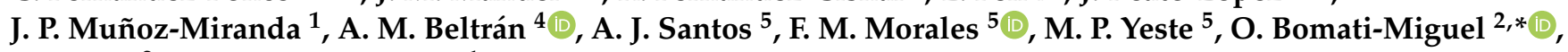 \\ R. Litrán ${ }^{2, *}$ and F. García-Cózar ${ }^{1}$
}

check for updates

Citation: Fernández-Ponce, C.;

Mánuel, J.M.; Fernández-Cisnal, R.; Félix, E.; Beato-López, J.;

Muñoz-Miranda, J.P.; Beltrán, A.M. Santos, A.J.; Morales, F.M.; Yeste, M.P.; et al. Superficial Characteristics and Functionalization Effectiveness of Non-Toxic Glutathione-Capped Magnetic, Fluorescent, Metallic and Hybrid Nanoparticles for Biomedical Applications. Metals 2021, 11, 383. https://doi.org/10.3390/met11030383

Academic Editor:

Houshang Alamdari

Received: 23 December 2020

Accepted: 22 February 2021

Published: 26 February 2021

Publisher's Note: MDPI stays neutral with regard to jurisdictional claims in published maps and institutional affiliations.

Copyright: (c) 2021 by the authors Licensee MDPI, Basel, Switzerland. This article is an open access article distributed under the terms and conditions of the Creative Commons Attribution (CC BY) license (https:// creativecommons.org/licenses/by/ $4.0 /)$
1 Department of Biomedicine, Biotechnology and Public Health, University of Cadiz and Institute of Biomedical Research Cadiz (INIBICA), 11003 Cadiz, Spain; ceciliamatilde.fernandez@uca.es (C.F.-P.); fecir80@gmail.com (R.F.-C.); juampmunoz@gmail.com (J.P.M.-M.); curro.garcia@uca.es (F.G.-C.)

2 Department of Condensed Matter Physics, Faculty of Sciences, IMEYMAT: Institute of Research on Electron Microscopy and Materials, University of Cádiz, Puerto Real, 11510 Cadiz, Spain; jose.manuel@uca.es (J.M.M.); eduardo.felix@uca.es (E.F.)

3 Departamento de Ciencias, Institute for Advanced Materials (INAMAT), Campus de Arrosadia, Universidad Pública de tgNavarra, 31006 Pamplona, Spain; juanjesus.beato@unavarra.es

4 Departamento de Ingeniería y Ciencia de los Materiales y del Transporte, Sevilla 41011, Spain; abeltran3@us.es

5 Department of Materials Science and Metallurgic Engineering, and Inorganic Chemistry, Faculty of Sciences, IMEYMAT: Institute of Research on Electron Microscopy and Materials University of Cadiz, Puerto Real, 11510 Cadiz, Spain; antonio.santos@gm.uca.es (A.J.S.); fmiguel.morales@uca.es (F.M.M.); pili.yeste@uca.es (M.P.Y.)

* $\quad$ Correspondence: oscar.bomati@uca.es (O.B.-M.); rocio.litran@uca.es (R.L.); Tel.: +34-667464426 (R.L.)

$\dagger$ Equal contribution.

Abstract: An optimal design of nanoparticles suitable for biomedical applications requires proper functionalization, a key step in the synthesis of such nanoparticles, not only for subsequent crosslinking to biological targets and to avoid cytotoxicity, but also to endow these materials with colloidal stability. In this sense, a reliable characterization of the effectiveness of the functionalization process would, therefore, be crucial for subsequent bioconjugations. In this work, we have analyzed glutathione as a means to functionalize four of the most widely used nanoparticles in biomedicine, one of which is a hybrid gold-magnetic-iron-oxide nanoparticle synthetized by a simple and novel method that we propose in this article. We have analyzed the colloidal characteristics that the glutathione capping provides to the different nanoparticles and, using information on the Z-potential, we have deduced the chemical group used by glutathione to link to the nanoparticle core. We have used electron microscopy for further structural and chemical characterization of the nanoparticles. Finally, we have evaluated nanoparticle cytotoxicity, studying cell viability after incubation with different concentrations of nanoparticles, showing their suitability for biomedical applications.

Keywords: functionalization; glutathione; surface modifications; colloidal stability; cytotoxicity; electron microscopy; magnetic nanoparticle; gold nanoparticle; quantum dot

\section{Introduction}

Advances in biomedicine require new experimental tools that enable manipulation of biomolecules, and the study of biological processes at the molecular and cellular level. For some decades now, nanoparticles (NPs) have been broadly used in biomedicine [1-4]. A large variety of NPs have been utilized for the development of applications in diagnostics [5,6], therapy [7,8], biomarking [9,10], drug delivery [11], etc. [12]. In this context, three groups of NPs are traditionally considered of the highest interest for biomedical 
applications, due to their properties: fluorescent NPs (quantum dots, QDs), magnetic NPs and pure metallic NPs.

The biomedical interest in light-emitting NPs resides in the fact that many biological techniques are based on the use of fluorometric probes for the identification of specific biological species. Therefore, unless genetic expression is desired, NPs such as QDs represent a successful alternative for biological markers, because of their advantageous features when compared to conventional organic fluorophores or fluorescent proteins, especially due to their size-dependent fluorescence. Light emission of these QDs can be tuned by controlling their size at nanometric level, which is an interesting characteristic for their use as biological probes in multicolor experiments [13-15].

In the case of magnetic NPs, applications for protein immobilization [16], as hyperthermia agents for cancer therapy [17,18], for drug delivery [19], and as contrast agents for magnetic resonance imaging (MRI) [1,20] have been proposed [21]. Particularly, iron oxides and especially magnetite $\left(\mathrm{Fe}_{3} \mathrm{O}_{4}\right)$ NPs are the most commonly used, due to their biocompatibility, low cytotoxicity and stability. MRI is a non-invasive clinical diagnostic technique with a high spatial resolution that is extensively used for anatomical imaging of soft body tissues [22]. However, this technique requires the use of contrast agents to enhance differences between damaged and normal tissues and is still lacking in technologies that allow for targeted identification of specific structures, such as tumors or infectious agents. Superparamagnetic iron oxide NPs are used in MRI as transverse relaxation time contrast agents, since they show excellent magnetic properties, in addition to their low toxicity and cytocompatibility $[23,24]$.

Among the purely metallic NPs and over the last two decades, Au NPs have been widely studied and applied in the field of biomedicine, especially in cancer diagnostics [25], tumor therapy by optical hyperthermia [26], biosensing [27], virus detection and regulation of cell function [28] as well as X-ray computed tomography (CT) contrast agents [1,29,30]. The biomedical interest of these NPs resides in their good biocompatibility, low inherent toxicity [31], facile synthesis, high biofunctionalization capability and in their unique optical properties due to their associated localized surface plasmon resonance (LSPR) [32]. For Xray $\mathrm{CT}$, contrast agents that allow for the display of internal tissue structures, are usually necessary, in order to enhance the image contrast and to provide additional functional information. Gold NPs can potentially improve the contrast provided by iodine contrast agents currently in use for cardiovascular imaging at clinically relevant energies (80-140 kV) [33].

Moreover, synthesis of hybrid NPs, such as structures constituted by a magnetic core covered by a gold shell, allows for the combination of features from both constituents, which enhances NP possibilities for specific biomedical applications. There is great interest in these hybrid NPs for therapies such as tumor treatment by hyperthermia, while they also constitute highly attractive materials for image based medical diagnostics [34]. Moreover, the latter frequently require a combination of data obtained from different complementary techniques in order to achieve accurate diagnoses [35] and thus these core-shell NPs may be simultaneously imaged by several techniques offering a promising alternative to the fabrication of multimodal contrast agents [36]. In this sense, different synthesis for hybrid magnetic iron oxide/gold NPs have previously been proposed, either naked, functionalized with capping agents, or by preparing separately magnetic iron oxide (magnetite or maghemite) and gold NPs and promoting a subsequent conjugation. Many of these procedures involve temperatures higher than $190{ }^{\circ} \mathrm{C}$ as well as the use of organic or hydrophobic solvents such as 1,2-hexadecanediol, 1-octadecene, etc. Thus, ligand exchange steps are required to obtain hydrophilic functionalized NPs.

However, NPs used for biomedical applications must meet some highly important requirements: they must display low cytotoxicity, colloidal stability, and availability for subsequent bioconjugation with other biomolecules. Surface functionalization is a common strategy not only to promote subsequent crosslinking between NPs and specific biological species, but also to minimize their cytotoxicity and unspecific binding. Functionalization of the NP surface with species containing bio-active terminal groups, such as amino or 
carboxylic groups, allows for subsequent linking with relevant biomolecules for targeted applications [37]. NP functionalization can also contribute to avoid aggregation and unspecific cellular uptake, minimizing accumulation in organs and/or phagocyte activation, thus maintaining a prolonged circulation time [38-40]. In any case, obtaining all the information available on the effectiveness of the functionalization process, via a comprehensive characterization, is crucial for further bioconjugations and biomedical applications.

The tripeptide glutathione (GSH) constitutes an interesting asset for functionalization, as it is a low-cost reagent that harnesses in its small frame three highly used moieties for bioconjugation, such as a thiol terminal group and also an amino and two carboxylic groups. In this sense, the present work follows previous ones, in which we presented the functionalization of different NPs with GSH [14,41,42]. In this work, we have utilized GSH to functionalize four types of NP: fluorescent CdTe QDs, iron oxide magnetic NPs, gold NPs and hybrid iron oxide magnetic-gold NPs, labeled in the text, as QD-GSH, MagGSH, Au-GSH and Au-Mag-GSH, respectively. Glutathione has been chosen because it is a small molecule with three different types of functional groups that can easily be used in order to bind other proteins such as streptavidine or antibodies. Furthermore, the thiol moiety readily binds to gold which is of interest for gold containing NPs. In this work, we have designed a simple and novel hydrophilic route to prepare hybrid goldiron oxide magnetic NPs with GSH in situ functionalization. These NPs are labeled as Au-Mag-GSH NPs. We have compared the results for these NPs with those obtained for different types of GSH functionalized NPs: gold, magnetic iron oxide and CdTe QDs. We have applied chemical synthetic routes based on reduction reactions, using sodium borohydride and performing an in situ functionalization with GSH. In the literature there are other examples of GSH-capped NPs $[43,44]$, but in all cases, the authors use different steps for the synthesis and perform an ex situ functionalization by a ligand exchange process, requiring, in some cases, the use of non-aqueous solvents. After a first characterization of the structural and physical properties of the NPs, we have evaluated the effectiveness of the functionalization process. With this aim, we have carried out a colloidal characterization, measuring the colloidal stability of the studied NPs at different $\mathrm{pH}$ values, different temperatures and different salinity, all fundamental features for biomedical applications. This study, combined with Fourier transform infrared (FTIR) spectroscopy, also provides information about the linking between NP and GSH. Depending on particle composition, GSH uses different terminal groups to link to the nanostructure. Moreover, we have combined electron microscopy-based structural and compositional nanoanalyses to obtain information about the composition and the thickness of the GSH layer surrounding the NPs. This characterization allows us to learn whether GSH has been properly linked to the NP surface thus functionalizing it. Finally, we have studied the cytotoxicity of the prepared NPs, analyzing cell viability after incubation of cells with different NP concentrations. The novelty of our work lies in the development of an easy and new synthetic method to prepare hybrid gold/iron oxide magnetic NPs, in situ functionalized with GSH. Our main objective is to deepen in the characterization of GSH functionalization and, in the knowledge of the colloidal characteristics of the resultant NP-based system, provide the basis for a material that could be successfully utilized in biomedical application.

In previous works [45], we showed an initial, mainly transmission electron microscopy (TEM)-focused, characterization of similarly prepared NPs but, due to their relevant biomedical properties, in this article we have deepened on their biomedical compatibility and surface capping both crucial for biomedical focused bioconjugation. Thus, here, we provide a new horizon for the design of GSH-capped gold/iron oxide magnetic NPs suited for biomedical applications and also expand in the mechanisms underlying GSH layer linkage, studying the effect that GSH capping causes to NP properties. For this purpose, different GSH NPs have been prepared and included in the study. Although GSH capped NPs have been previously studied, this work presents a deep characterization of their colloidal properties, the functionalization mechanism and its influence on cytotoxicity. 


\section{Materials and Methods}

\subsection{Chemicals}

The following chemical reagents were utilized in this work: tetrachloroauric acid $\left(\mathrm{HAuCl}_{4}\right)$, cadmium chloride $\left(\mathrm{CdCl}_{2}\right)$, sodium borohidrure $\left(\mathrm{NaBH}_{4}\right)$, tellurium powder, sodium hydroxide $(\mathrm{NaOH})$, iron $(\mathrm{III})$ chloride $\left(\mathrm{FeCl}_{3}\right)$, sodium hydroxide $(\mathrm{NaOH})$, diethylene glycol, cysteamine (CYS) and reduced glutathione (GSH). All these reagents were of analytic grade, purchased from Sigma-Aldrich (current "Millipore-Sigma", Burlington, MA, USA) and used as received. Milli-Q®(MQ) water was used for all experiments.

\subsection{Synthesis of Glutathione-Capped Nanoparticles (GSH-NPS)}

Based on our previous works, we have designed three rapid chemical routes to obtain small GSH-capped gold, magnetic iron oxide, and CdTe QDs [14,42]. In all these routes, we have promoted a reduction reaction, and we have used GSH to functionalize the NP surface. To synthetize the GSH-capped hybrid gold-iron oxide magnetic NPs, we have developed, in this work, a novel, original method. A diagram of the formation of these GSH-capped NP is shown in the graphical abstract.

\subsubsection{GSH-Capped Gold NPs}

Briefly, we started from $20 \mathrm{~mL}$ of an aqueous solution $0.01 \mathrm{M} \mathrm{HAuCl}_{4}$ and $0.026 \mathrm{M} \mathrm{GSH}$. The mixture was vigorously stirred for 30 min under a nitrogen atmosphere. $\mathrm{A} \mathrm{NaBH}_{4}$ aqueous solution was added dropwise, in a 1:5 $\mathrm{HAuCl}_{4} / \mathrm{NaBH}_{4}$ molar ratio. After 30 additional min, the red wine colored solution was stored at $8{ }^{\circ} \mathrm{C}$ protected from light. The code $\mathrm{Au}-\mathrm{GSH}$ is used for this type of NPs. In this case, the $\mathrm{Au}^{3+}$ ions, introduced as $\mathrm{HAuCl}_{4}$, are reduced by the $\mathrm{NaBH}_{4}$. Although GSH could act as a reducing agent, the reaction would be slower than with a strong reducing agent, such as $\mathrm{NaBH}_{4}$, that will promote a fast nucleation, leading to more homogeneous and smaller NPs.

\subsubsection{GSH-Gapped Iron Oxide Magnetic NPs}

In this case, we designed a preparation method based on the thermal decomposition of an iron salt, in the presence of GSH, to functionalize the NP, thus controlling its final size.

The synthesis of GSH-iron oxide magnetic NPs was carried out in two steps. The first consisted of the preparation of a $\mathrm{NaOH}$ solution that will be used as oxidizing agent for the co-precipitation of iron and formation of iron oxide. This solution was prepared by heating $20 \mathrm{~g}$ of $\mathrm{NaOH}$ and $20 \mathrm{~mL}$ of diethylene glycol at $120^{\circ} \mathrm{C}$ for one hour. The resulting solution is stored at $70{ }^{\circ} \mathrm{C}$ for subsequent utilization.

The second step constituted the main thermal decomposition and co-precipitation reaction. $0.55 \mathrm{~g}$ of $\mathrm{FeCl}_{3}$, used as iron precursor, have been solved into $15 \mathrm{~mL}$ of diethylene glycol, which is used as solvent. The solution obtained was placed in a $500 \mathrm{~mL}$ threenecked flask, where the GSH, used as capping agent, was added. The GSH:Fe molar ratio was 1:9. The solution was heated at $200{ }^{\circ} \mathrm{C}$ under reflux in a nitrogen atmosphere, in order to promote the thermal decomposition of iron salts. After heating for $30 \mathrm{~min}$, a previously prepared $8 \mathrm{~mL} \mathrm{NaOH}$ solution was added, to induce the formation of iron oxide. Once $\mathrm{NaOH}$ was added, the total solution was heated for $10 \mathrm{~min}$, keeping the temperature at $200^{\circ} \mathrm{C}$. After cooling, the obtained solution was filtered three times (using a $0.1 \mu \mathrm{m}$ Millipore membrane), after precipitation with ethanol, in order to eliminate an excess of precursor species, and/or, residues from the reaction. The $\mathrm{Fe}^{3+}$ cations, introduced as $\mathrm{FeCl}_{3}$, were totally or partially reduced by the diethylene glycol, giving rise to the formation of either magnetite $\left(\mathrm{Fe}_{3} \mathrm{O}_{4}\right)$ or maghemite $\left(\gamma-\mathrm{Fe}_{2} \mathrm{O}_{3}\right)$, both iron-oxide magnetic phases equally appropriate for the objective of this work. The high GSH affinity for the NP surface led to GSH-functionalized magnetic NPs (Mag-GSH) as a black powder with magnetic character. 


\subsubsection{GSH-Capped Gold/ $\mathrm{Fe}_{3} \mathrm{O}_{4} \mathrm{NPs}$}

Starting from the Mag-GSH NPs previously prepared, we designed a method to easily prepare GSH capped gold/iron oxide magnetic NPs. In this novel synthetic route, we use the capability of GSH to link iron oxide and gold simultaneously. Briefly, $0.05 \mathrm{~g}$ Mag-GSH, prepared as descried above, were dispersed in $1 \mathrm{~mL}$ of MQ water while stirring for $1 \mathrm{~h}$. Then, an aqueous solution containing $0.1 \mathrm{~g} \mathrm{HAuCl}_{4}$ was added. After $8 \mathrm{~h}$ of vigorous stirring, GSH was added in excess while stirring for one additional hour. The total synthesis was conformed in three simple steps. The first step was just the Mag-GSH preparation. In the second step, free terminal GSH moieties in Mag-GSH NPs bound to gold when we added the $\mathrm{Au}^{3+}$ precursor. In this case, a slow reduction of $\mathrm{Au}^{3+}$ by the same GSH used as capping agent was promoted, in order to avoid the formation of separated gold NPs, a process that could be induced by the addition of $\mathrm{NaBH}_{4}$. During the last step, GSH was added in excess and linked to the final hybrid iron oxide-gold NPs. The colloidal solution was brought under magnetic decantation, in order to eliminate the iron oxide magnetic NPs which did not contain gold. As a result, a brown-pink and still magnetic colloidal solution was obtained. The solution could be filtered and washed, obtaining a magnetic, brown-black powder. As in the case of Mag-GSH, these NPs can easily be dissolved in an aqueous solution due to the hydrophilic character conferred by GSH. A schematic graphic of this particular synthesis is shown in Figure 1.
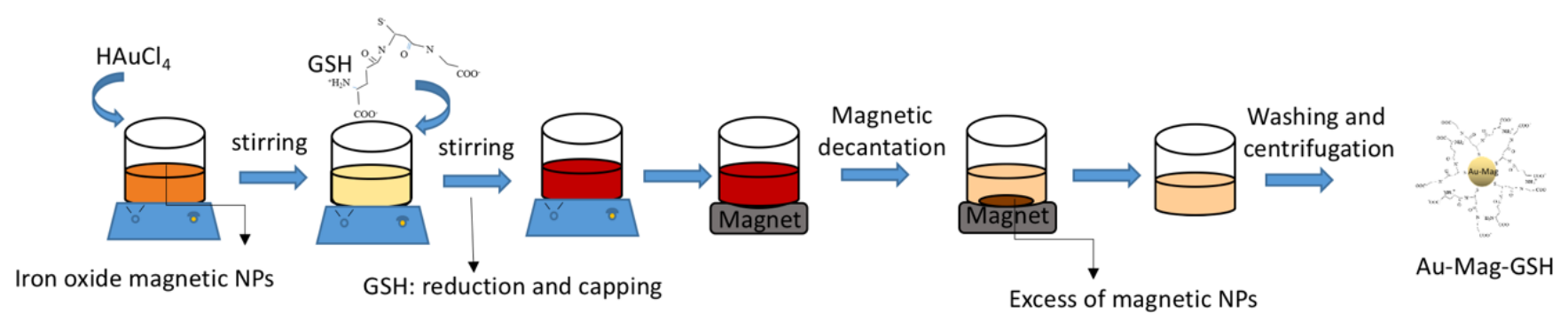

Figure 1. Diagram of the glutathione (GSH)-capped hybrid gold/iron oxide magnetic nanoparticles (NPs).

\subsubsection{GSH-Capped QDs}

Synthesis of GSH-CdTe QDs was carried out by a co-precipitation reaction, using $\mathrm{CdCl}_{2}$ as cadmium precursor and $\mathrm{NaHTe}$ as tellurium precursor in the presence of $\mathrm{GSH}$. For this purpose, $0.19 \mathrm{~g} \mathrm{GSH}$, used as a stabilizing agent, and $0.046 \mathrm{~g} \mathrm{CdCl}_{2}$, used as the cadmium precursor, were dissolved in $100 \mathrm{~mL}$ MQ water. In order to promote formation of $\mathrm{Cd}^{2+}$-GSH complexes at the QD surface, the $\mathrm{pH}$ was adjusted to 8 by dropwise addition of a $1 \mathrm{M} \mathrm{NaOH}$ solution, under vigorous stirring. The obtained solution was placed in a $500 \mathrm{~mL}$ three-necked flask, where $2 \mathrm{~mL}$ fresh $\mathrm{NaHTe}$ (previously prepared by reducing tellurium powder with $\mathrm{NaBH}_{4}$ in a nitrogen atmosphere) was added. The $\mathrm{Cd}^{2+}$ :NaHTe:GSH molar ratio was 4:1:10. The solution was heated under reflux in a nitrogen atmosphere. The final QD size can be controlled by increasing temperature and/or reaction time, ranging from 90 to $145^{\circ} \mathrm{C}$ and 90 to $360 \mathrm{~min}$, respectively.

The different aqueous aliquots, containing the GSH-CdTe QDs, were cooled and filtered threefold (using a $0.1 \mu \mathrm{m}$ Millipore membrane), after precipitation with a mixture of acetone and ethanol, in order to eliminate the excess of precursor species and/or, residues from the reaction. As a result, we obtained CdTe QDs capped with GSH molecules (QD-GSH) that can be easily dispersed in and aqueous solution due to the hydrophilic character conferred by GSH.

\subsection{Characterization Techniques}

\subsubsection{Structural and Chemical Analyses}

The identification of GSH-NPs has been carried out through structural and chemical techniques, associated with transmission and scanning-transmission electron microscopy, 
or (S)TEM. The general view, shape and atomic structure of the materials were observed using conventional bright field (BF-TEM), high-resolution (HRTEM) and high-angle annular dark field STEM (HAADF-STEM). Also, a corrected probe HAADF in high-resolution mode (HR-HAADF) was utilized for the sample Au-GSH, yielding images in which these crystals were easily distinguishable (due to the high difference of atomic numbers between the gold in the NP and the carbon in the supporting material, which leads to a high contrast in the image intensity for both structures) and simultaneously show atomic columns. Regarding chemical composition, energy-dispersive X-ray spectroscopy (EDX) was applied, so the structural information from imaging techniques is complemented, allowing an unequivocal identification of the NP and revealing the GSH shell. All these (S)TEM techniques were carried out using two STEM microscopes: a double-aberration corrected TITAN ${ }^{3}$ Themis and a Talos F200X ("ThermoFisher Scientific", Waltham, MA, USA), both operating at a $200 \mathrm{kV}$ accelerating voltage. In order to observe the different materials under the electron beam, several samples were prepared by depositing $10 \mu \mathrm{L}$ of an NP colloidal solution, drop-casted onto a holey-carbon coated $\mathrm{Cu}$ grid for TEM and dried for five hours. Alternatively, some samples, for Mag-GSH, were prepared by imbibing a grid over a dry amount of material, obtaining a sample with no significant difference in the observed NP state of aggregation when deposited on the sample grid. For all magnetic materials, a magnet was placed near to the TEM grid before inserting the sample in the electron microscope in order to remove the biggest NP clusters, which could be attracted by the magnetic lenses of the microscopes.

FTIR was utilized in order to obtain information about the linking between GSH and the NP surface. Experiments were recorded with Bruker Alpha System Spectrophotometer (KBr wafer technique), using the same quantity of sample in all measurements.

\subsubsection{Colloidal Characterization}

Dynamic light scattering (DLS) was utilized in order to measure NP hydrodynamic size. Measurements were carried out at 25,37 and $40{ }^{\circ} \mathrm{C}$, using a Malvern Zetasizer NanoZS ("Malvern Instruments", Worcestershire, UK), with a $1 \mathrm{~cm}$ path cell. This equipment also allows for the Z-potential to be measured which evaluate colloidal stability in solution, as well as NP surface charge.

\subsubsection{Optical and Magnetic Properties}

LSPR of the Au-GSH nanostructure was evaluated studying the ultraviolet-visible (UV-Vis) absorbance of each NP colloidal solution. Absorption spectra were acquired with a Lambda 19 Perkin Elmer spectrophotometer ("Perkin Elmer", Waltham, MA, USA). The position and profile of the LSPR band provide information about NP dispersion and allow for the estimation of NP average sizes.

Photoluminescence (PL) excitation and emission spectra were recorded in a PTI Quantamaster fluorometer using a Xenon arc Lamp at $150 \mathrm{~W}$ and a computer controller QuadraScopic monochromator.

Magnetic properties of magnetic-iron-oxide NPs were analyzed by measuring magnetization curves at room temperature in a high-sensitive Magnetic Faraday Balance ("Oxford Instruments", Abingdon, U.K.), applying magnetic fields up to 0.6 T.

\subsubsection{Cytotoxicity Assay and Cell Culture}

Jurkat cells ("American Type Culture Collection", Manassas, VA, USA) were cultured at $37^{\circ} \mathrm{C}$, in a $5 \% \mathrm{CO}_{2}$ atmosphere, in Dulbecco's Modified Eagle's Medium (DMEM) containing $2 \mathrm{mM}$ L-glutamine, $10 \mathrm{mM}$ Hepes, 10\% $(v / v)$ heat-inactivated fetal bovine serum (FBS), 1\% $(v / v)$ non-essential amino acids (NEAA), 1\% ( $v / v)$ sodium pyruvate, $50 \mu \mathrm{M}$ 2-mercaptoethanol, $100 \mathrm{U} / \mathrm{mL}$ penicillin and $100 \mu \mathrm{g} / \mathrm{mL}$ streptomycin (all from "Life Technologies", Carlsbad, CA, USA). 


\subsubsection{Cytotoxicity and Cell Viability.}

We cultured $5 \times 10^{5}$ Jurkat cells cells in a 48-well plate, in the absence or presence of NPs at the indicated concentrations. Cell viability was analyzed $24 \mathrm{~h}$ after addition of NPs by a As previously commented, one key property (MTT)-based assay as previously described. Briefly, MTT reactant (thiazolyl blue tetrazolium bromide, TOX1-1KT, "Millipore-Sigma", Burlington, MA, USA) was added to the cells in a 1:10 ratio (MTT solution/culture medium) and incubated during $3 \mathrm{~h}$ at $37^{\circ} \mathrm{C}$. Then, formazan crystals, formed inside viable cells, were dissolved by adding MTT Solubilization Solution M-8910, ("Millipore-Sigma", Burlington, MA, USA) at 1:2 with vigorous pipetting. Optical density at $570 \mathrm{~nm}$ was evaluated to quantify the amount of formazan crystals, which is proportional to the number of viable cells (background absorbance was measured at $690 \mathrm{~nm}$ and subtracted from the $570 \mathrm{~nm}$ measurement). Viability of cell in the presence of NPs was compared to untreated controls (considered 100\% viability). Media in the absence of cells was used as blank; while cells, cultured in the presence of $10 \%$ dimethyl sulfoxide (DMSO), was used as control for decreased cell viability, being always below $10 \%$. Statistical analyses were performed using the Statgraphics software ("Statpoint Technologies", Warrenton, VA, USA). Significances were determined by using analyses of variance (ANOVA) and multiple range tests.

\section{Results}

As previously commented, in order to determine the potential of the presented NPs for biomedicine applications, it is required to previously study their structural properties, their optical behavior, as well as additional features such as colloidal stability at physiological values of salinity and $\mathrm{pH}$, colloidal stability at temperatures ranging from 35 to $40^{\circ} \mathrm{C}$ and cytotoxicity.

\subsection{Structural Features and Physical Properties}

Figure 2 shows results on the size and optical properties of Au-GSH samples, as well as an overview on the state of aggregation of the NPs when deposited on the holed carbon grid. A general view of these particles can be observed in Figure $2 a$, which shows a HAADF-STEM micrograph. This technique is ideal for imaging gold-based particles, due to the high atomic number of gold, which produces a high contrast with the carbon support in HAADF images. This micrograph reveals the formation of relatively small and well-dispersed NPs with nearly round shape. Figure $2 b$ shows the NP size distribution obtained from more than 300 NPs in this and similar images. From this histogram, it can be affirmed that particle size is homogeneous, with an average diameter of $3.8 \mathrm{~nm}$ (see Table 1, in which particle diameter for all materials are collected), obtained from the fitting of this distribution to a Gaussian function. In any case, although this type of images shows, with a good contrast, the size and shape of the particles, hinting on the high atomic number of its constituent material, the exact chemical composition of the NPs is not determined with this technique. The presence of gold in this nanostructure can be confirmed by the UV-Visible absorption spectrum of the colloidal solution (Figure 2c). The inset in the figure is a photograph of the obtained red-wine colored colloidal solution. The spectrum shows the characteristic $520 \mathrm{~nm}$ LSPR absorption band, indicating the presence of small gold NPs. Position and width of LSPR bands give information on NP size and size homogeneity. The Au-GSH LSPR band appears as a shoulder, showing a wide profile which can be a consequence of the small average size of these NPs. The high number of surface atoms in small NPs increases the damping of oscillating electrons at conduction bands. This effect can be even higher for small thiol-capped NPs, due to the loss of itinerancy of electrons involved in Au-S bonding [46]. This ligand-metal charge transfer transition, produced due to the capping, can contribute to the relatively strong emission bands, in the blue range of the spectra, for the Au-GSH NPs, as shown in Figure 2d, where the PL emission spectra, recorded for the Au NPs colloidal solution (exciting at $317 \mathrm{~nm}$ ), is shown. This emission could be an interesting feature for the use of this NPs for biomarker applications [42]. 
a
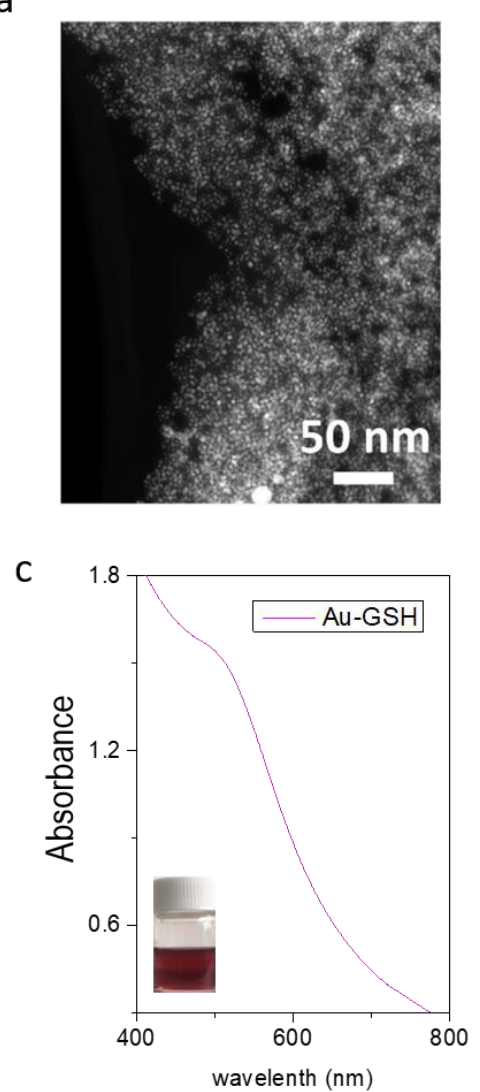

b

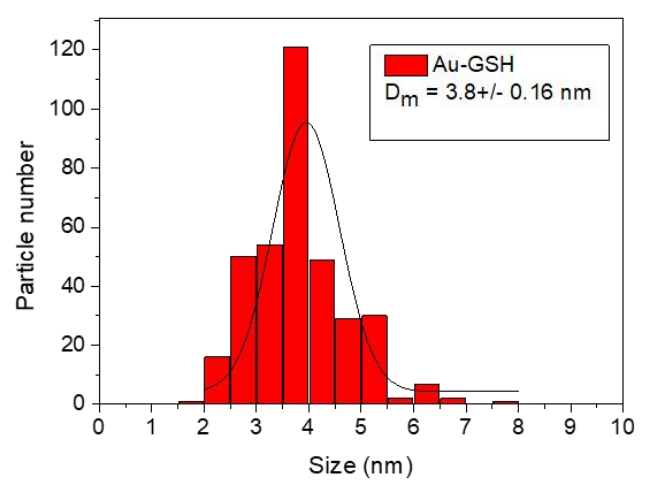

d

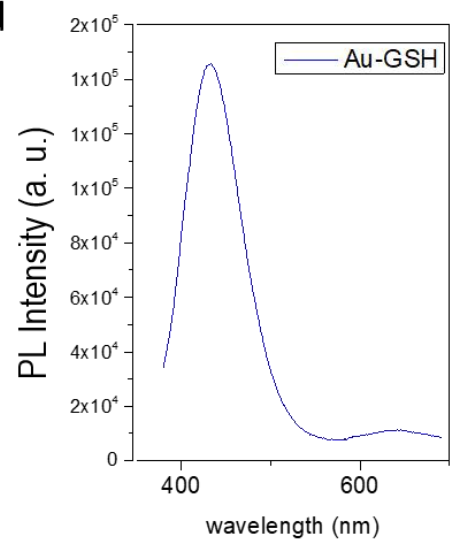

Figure 2. High-angle annular dark field (HAADF) image (a), as well as the histogram displaying particle size distribution (b) of Au-GSH NPs. (c) UV-Visible absorption spectrum and PL emission spectrum (d) of Au-GSH NPs. The inset in Figure 2c shows a digital photo image of an Au-GSH colloidal solution.

Table 1. Average diameter obtained via transmission electron microscopy (TEM), dynamic light scattering (DLS) hydrodynamic diameter and polydispersity index (PDI).

\begin{tabular}{cccc}
\hline Sample & $\begin{array}{c}\text { TEM Average Size } \\
(\mathbf{n m})\end{array}$ & $\begin{array}{c}\text { DLS Average Size } \\
(\mathbf{n m})\end{array}$ & PDI (\%) \\
\hline Au-GSH & $3.8 \pm 0.2$ & $6.2 \pm 1.2$ & 0.2 \\
Mag-GSH & $6.1 \pm 0.4$ & $12.7 \pm 1.2$ & 0.2 \\
QD-GSH & $2.9 \pm 0.3$ & $32.6 \pm 1.3$ & 0.1 \\
Au-Mag-GSH & $6.9 \pm 0.4$ & $42.3 \pm 1.3$ & 0.2 \\
\hline
\end{tabular}

Figure 3 shows results obtained from the optical and structural characterization of Mag-GSH samples. A general view of the NPs is showed in Figure 3a. The BF-TEM micrograph reveals the obtained particle distribution for this preparation, less dispersed as in the case of gold NPs, most probably due to the small clustering promoted by magnetic interactions. Figure $3 \mathrm{~b}$ displays and histogram showing the particle size distribution for this sample. Fitting of these results to a Gaussian function reveals a NP average size of $6.1 \mathrm{~nm}$ (Table 1). Figure 3c shows the magnetization curve, i.e., applied field versus magnetization, at room temperature. This curve shows the magnetic behavior of these NPs [47]. The inset in Figure 3c shows the response of Mag-GSH NPs (flask on the left) to a permanent magnetic field, applied using a permanent magnet adjacent to the flask that contains the NP colloidal dispersion. 
a

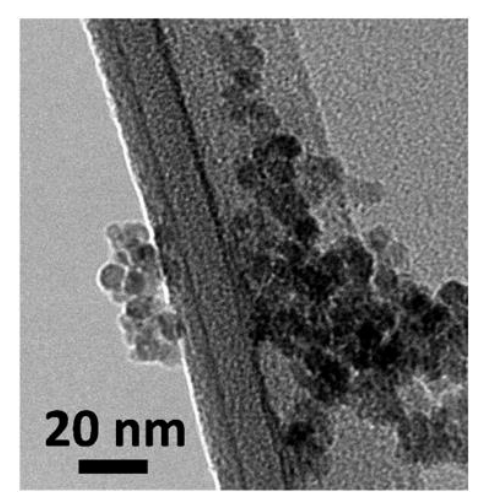

b

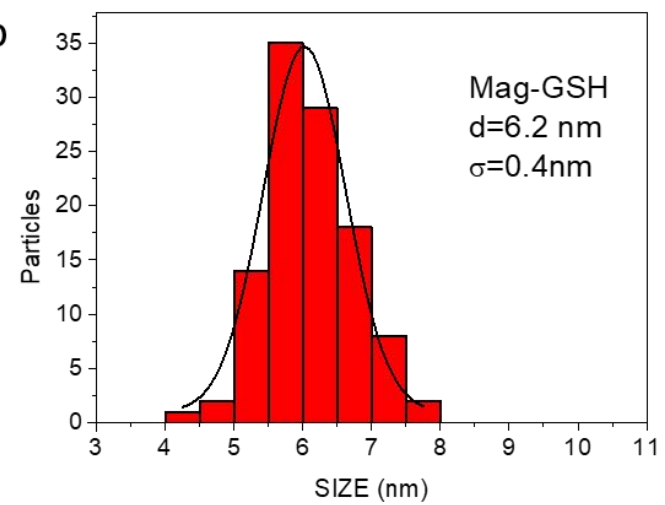

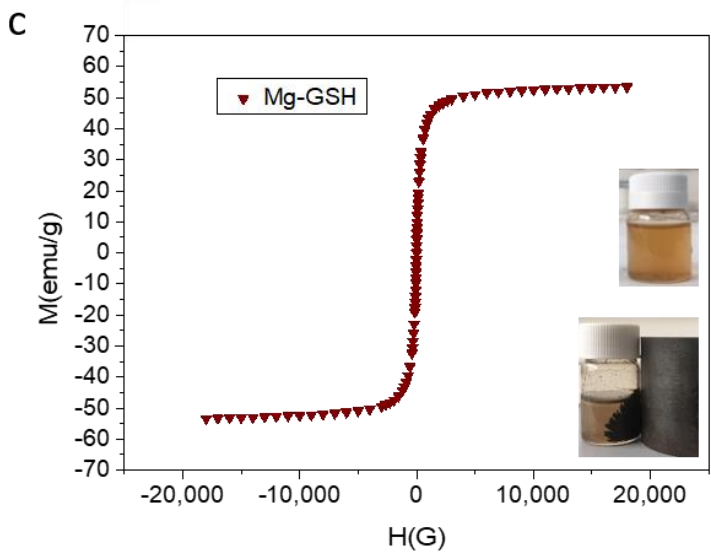

Figure 3. Bright field TEM (BF-TEM) micrograph (a), as well as the histogram displaying the particle size distribution (b) of Mag-GSH NPs. Magnetization curve of Mag-GSH NPs (c). The inset in this figure shows a digital photo image of Mag-GSH NPs placed in the absence and presence of a magnet located close to the vials.

Figure 4 shows a general view and optical features of QD-GSH NPs. Figure 4 a corresponds to a HAADF-STEM image of an accumulation of these QD-GSH NPs. In this case, we can observe the presence of extremely small NPs immersed in the GSH capping. The histogram corresponding to these NPs is shown in Figure $4 \mathrm{~b}$. By fitting these results to a Gaussian function, an average NP diameter of $2.9 \mathrm{~nm}$ is obtained (Table 1). As we have previously commented, NP size is especially important for their optical properties, determining the wavelength of their emission, which is especially important in this case, since QD functionality in biomedical applications rely on their fluorescence. In this sense, the proposed synthesis allows control of NP final size and thus, its PL emission. Specifically, Figure 4c shows PL emission spectra obtained from the sample in Figure 4a (with average size reflected in Figure 4b), labelled as QD-GSH1 and prepared at a synthesis temperature of $90{ }^{\circ} \mathrm{C}$ for $30 \mathrm{~min}$. Two additional QD-GSH samples of bigger average sizes (QD-GSH2, prepared at $90^{\circ} \mathrm{C}$ for $3 \mathrm{~h}$, and QD-GSH3, heating during $3 \mathrm{~h}$ at $90^{\circ} \mathrm{C}$ and one more hour at $110^{\circ} \mathrm{C}$ ) are also included. As NP size increases (sizes for QD-GSH1, QD-GSH2 and QD-GSH3 are $2.9 \mathrm{~nm}, 3.6 \mathrm{~nm}$ and $3.9 \mathrm{~nm}$, respectively), a red-shift in the emission peak maximum is observed, illustrating wavelength size-dependency of the emission. The photograph of colloidal solutions of these three samples, under UV radiation, is added to this figure, as an inset. As it can be observed from both, PL spectra and photograph of the colloidal solutions; by changing just a few nanometers in size, we can tune the emission color from blue to red.

Regarding hybrid Au-Mag-GSH NPs, one of the first and important results to be confirmed after their preparation was that each nanostructure is indeed formed by both iron oxide and gold. Taking into account that the synthesis has been initiated by heterogeneous nucleation of iron oxide seeds, the gold phase necessarily has to be located around the 
iron oxide phase. In this sense, a relevant measurement is shown in Figure 5a, namely the comparison of UV-Vis absorption spectra for the colloidal solutions of Au-GSH and AuMag-GSH samples. Both colloids, display the LSPR absorption band (associated to gold), however, the hybrid Au-Mag NP curve has a pronounced red-shift, from $560 \mathrm{~nm}$ to $520 \mathrm{~nm}$. According to previous reported results, this red-shift can be attributed to the reduced electron deficiency of Au NPs caused by the interfacial communication between $\mathrm{Au}$ and magnetic-iron-oxide [48]. Therefore, these results reveal the formation of a hybrid NP, containing gold and magnetic-iron-oxide. The Au-Mag-GSH spectrum, shows a second and less intense near-infrared (NIR) absorption band that could be attributed to the presence of anisotropic NPs or even to iron oxide-gold interactions in the hybrid structure. This characteristic can be of interest in phototherapy applications $[49,50]$. A photograph of the Au-Mag-GSH colloidal solution is also shown in this figure (see inset image in Figure 5a). The spectra have not been normalized because the determinant parameters are the LSPR band position, the wavelength at which the LSPR is observed and the shape and width of the band. A BF-TEM micrograph of these particles is shown in Figure 5b, revealing the formation of homogeneously shaped structures, without the presence of clusters as dense as those observed for Mag-GSH. NP size distribution, and its fitting to a Gaussian function is shown in Figure 5c, showing an average particle size of $6.9 \mathrm{~nm}$ (Table 1).

a

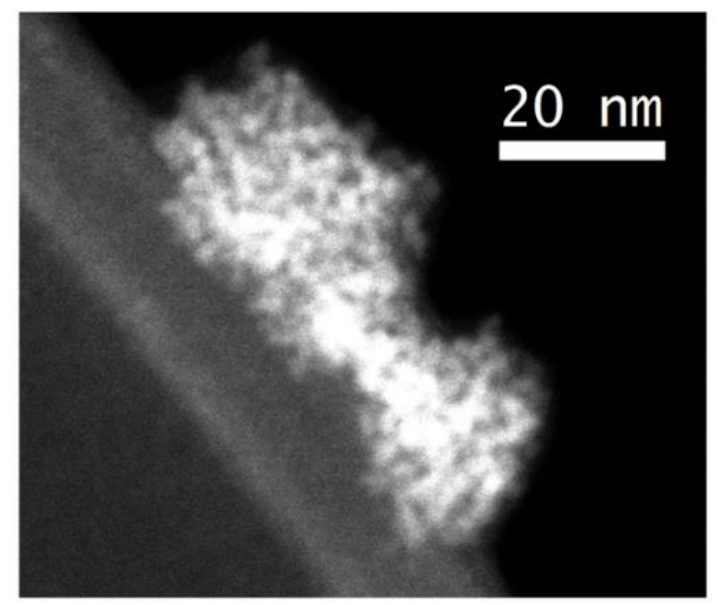

b

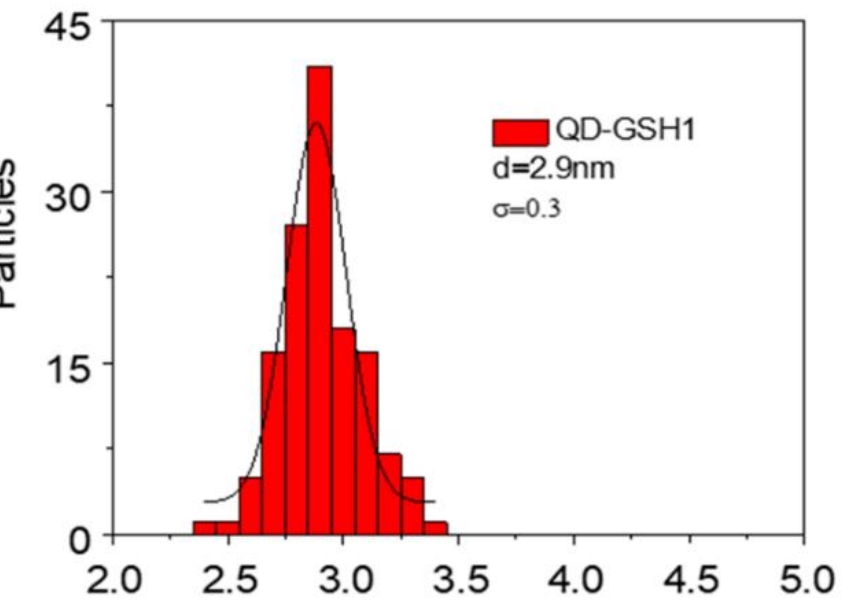

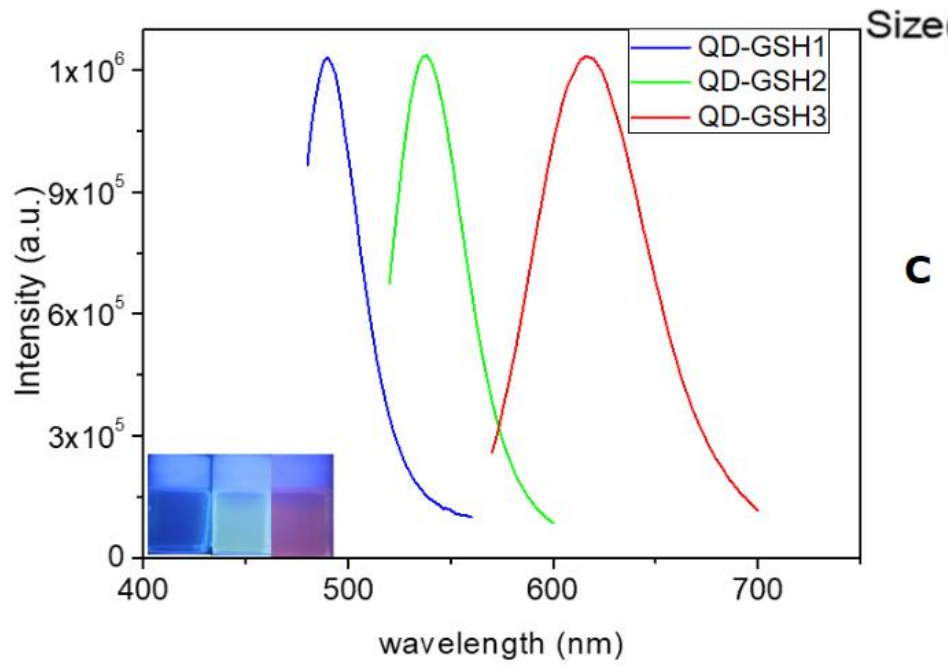

Figure 4. HAADF image (a), as well as the histogram displaying particle size distribution (b) of quantum dot (QD)-GSH1 NPs. (c) PL-emission spectra for QD-GSH1 (blue) as well as for QD-GSH2 (green) and QD-GSH3 (red), with higher average size. The inset in this figure shows a digital photo image of the three QD-GSH colloidal solutions used in this figure, under UV irradiation. 


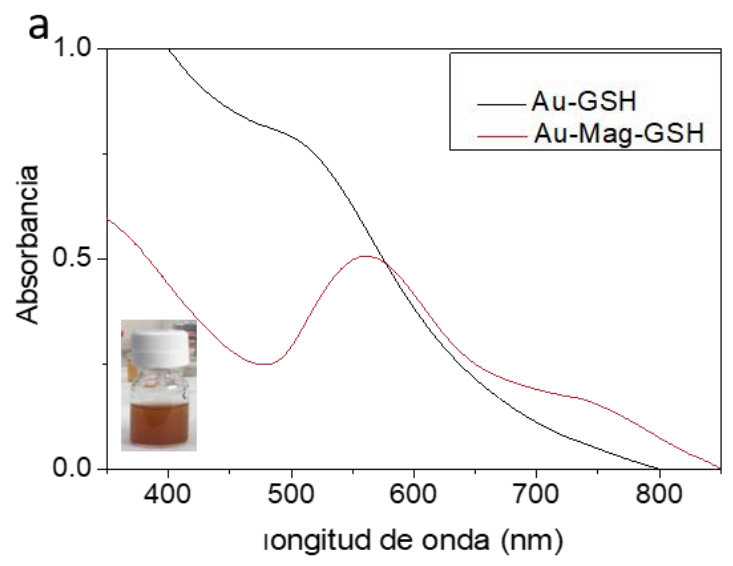

b

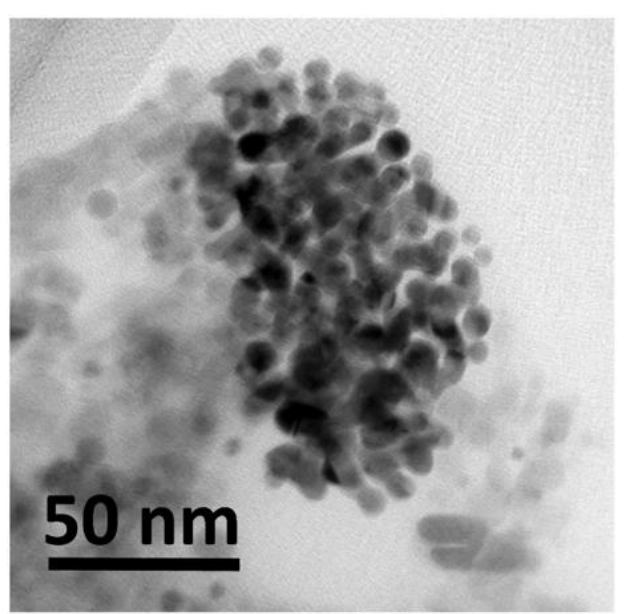

C

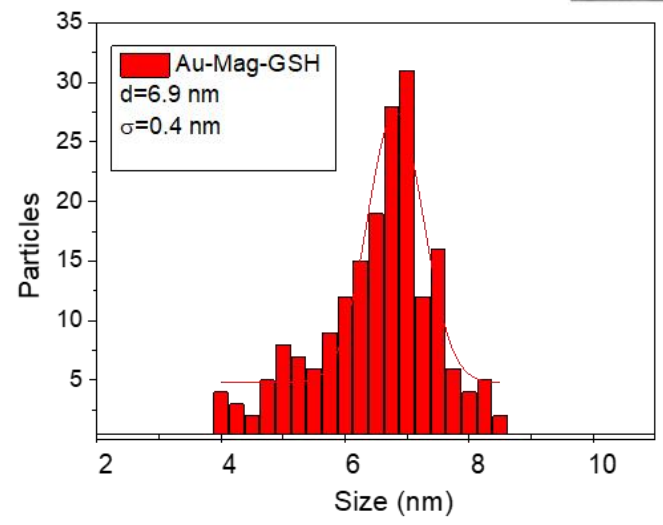

Figure 5. Ultraviolet (UV)-visible spectra for Au-GSH and Au-Mag-GSH colloidal solutions (a); TEM micrograph of Au-Mag-GSH (b) and its corresponding histogram displaying NP size distribution (c).

Figure 6 summarizes the main (S)TEM results on the structure and composition of the different GSH-capped NP samples. At this point, it is worth mentioning that the study via electron beam-based techniques of these materials produced a large amount of data and information. Therefore, more details on the (S)TEM characterization can be found elsewhere [45]. Figure 6a,b show respectively HRTEM and HR-HAADF micrographs, for Au-GSH NPs taken from the same region. The comparison of these modes, for the same NP, not only allows visualization of the crystalline structure, but also indicates the chemical homogeneity of such structure. Also, it is possible to visualize (Figure 6a) a thin amorphous layer (GSH) covering the NP core. Images such as the ones in Figure 6a,b allow measuring inter-planar distances of 2.17 and $2.38 \AA$, which respectively agree well with the spacing between $\{002\}$ and $\{111\}$ families of atomic planes for cubic Au. These values of inter-planar atomic distances have been obtained using the fast Fourier transform, FFT, (Figure 6a, inset) of a large enough region of the NP that show a crystalline structure with the same orientation. Using a generic diffraction software [51] to compare the experimental FFT with a simulated diffraction diagram, the zone axis can be identified and the diffraction peaks indexed. Note that FFT data are equivalent to electron diffraction diagrams when in Fourier condition, as is the case for the microscope optics. Similar analyses (not shown here) also revealed that crystalline structures were formed, in all samples and for each type of NP, corresponding to the expected elements and compounds. 

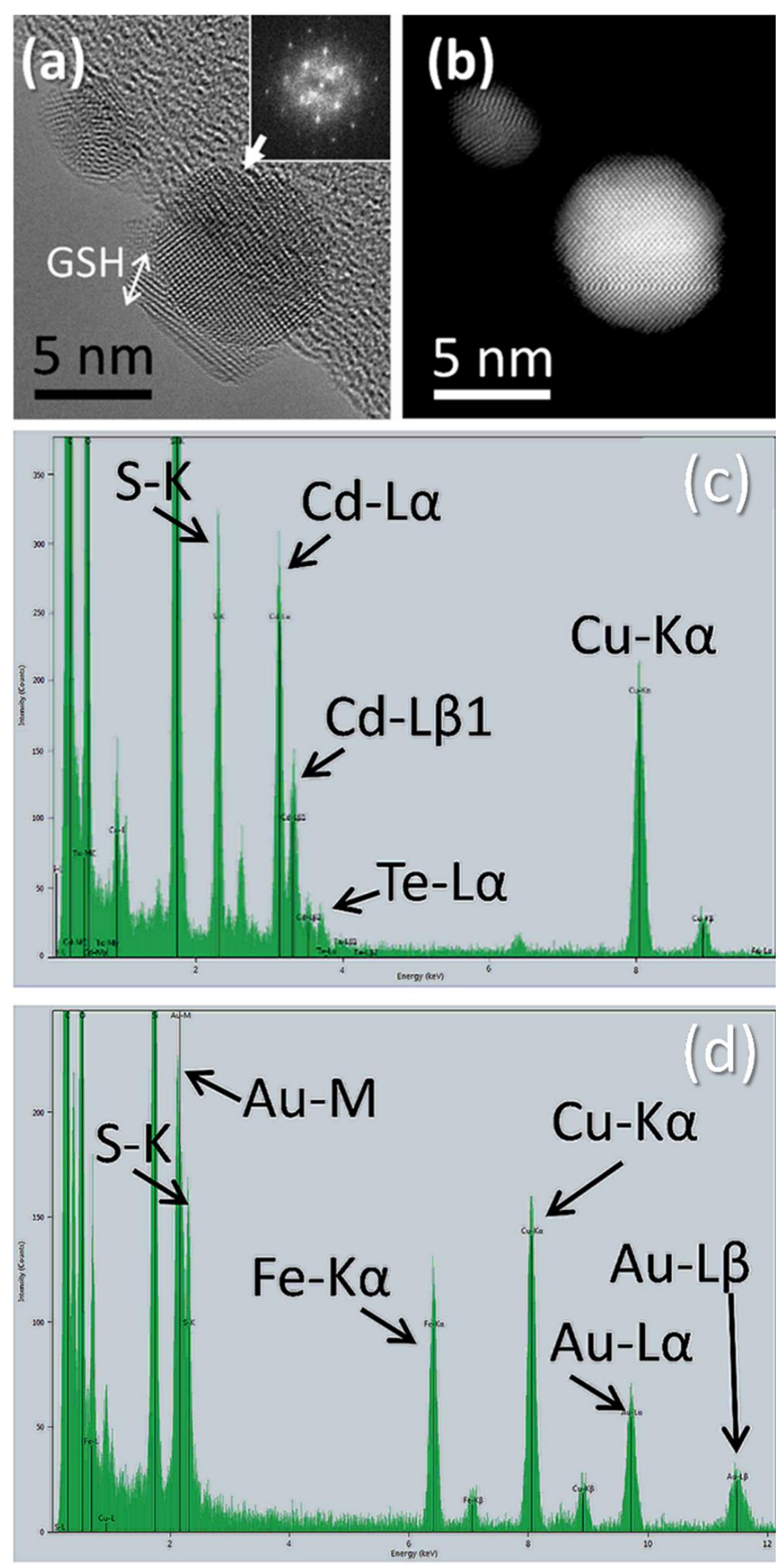

Figure 6. High-resolution TEM (HRTEM) (a) and HR-HAADF (b) images of NPs in sample Au-GSH. Punctual energy-dispersive X-ray (EDX) spectrum from QD-GSH (c) and from Au-Mag-GSH (d) NPs. 
Compositional sensitive STEM techniques, such as EDX, can complement these results, allowing analyze the uniformity of the GSH layer. In this sense, we have utilized the sulfur (S) peak associated to the K-level transition in order to detect GSH. Figure $6 \mathrm{c}$ presents an EDX-punctual spectrum for a NP of the same sample. Such a spectrum is taken from the area defined by the scanning STEM probe, which is estimated to correspond to $5 \mathrm{~nm} \times 5 \mathrm{~nm}$. Thus, the spectrum corresponds, approximately, to a region spanning, the size of one NP. Note, that other expected peaks, such as the ones associated to carbon and oxygen, also presented in the spectrum, are mostly due to both the carbon support and organic residual particles in the electron microscopy grid. In this sense, the copper signal, visible in all spectra is produced by the copper in such grid. In a previous work [45], EDX-maps for the different elements were presented, so a more comprehensive view of the STEM study for these samples can be obtained from this communication. Note that EDX results, such as those shown in Figure $5 \mathrm{f}$ from the study presented in reference 45, only indicate the iron-containing composition of the NP. The affirmation that the NP contains iron oxide would need further evidence. On the other hand, Figure $6 \mathrm{~d}$ presents the EDX punctual spectrum of isolated NPs from a sample of Au-Mag-GSH. All the main studied elements were found, revealing a hybrid structure in a large amount of NPs.

Base on all these results, it can be affirmed that the synthetic routes utilized to prepare the different NPs lead to the formation of small NPs, with homogeneous size distribution, a crystalline structure and a homogeneous composition, both for the core and for the polymer GSH covering layer. The in situ functionalization with GSH probably contributes to this morphological feature. These NPs show suitable characteristics for different biomedical applications, depending on the core composition. However, the use of NPs as potential tools in biomedicine, requires potential capability to be bioconjugated with biological species. The effectiveness of the functionalization process is, in any case, fundamental for subsequent bioconjugations.

\subsection{Colloidal Stability and Superficial Properties}

\subsubsection{Hydrodynamic Size}

To evaluate the colloidal stability of the different NPs, DLS experiments have been carried out. Figure 7 shows the DLS diameter distribution (represented in number) obtained for the different GSH NPs. Each distribution has been fitted to a log-normal function, also shown in the graphics. Colloidal average diameters obtained from these fittings, as well as the PDI (polydispersity index) are shown in Table 1.

All NPs show a relatively narrow size distribution with colloidal average sizes smaller than $50 \mathrm{~nm}$, and with a relatively low polydispersity index (PDI in Table 1). As expected, all NPs show DLS hydrodynamic sizes bigger than those obtained from TEM, since this last technique measures mostly the crystalline volume of the particles (as explained before, in most particles, the polymer layer is veiled by the carbon support in the images). This effect is not only due to the GSH layer, but also to the presence of extra hydrate layers in aqueous medium. However, the similar sizes found from both TEM and DLS, in the case of Au-GSH, reveals the high colloidal stability of these NPs. Also, in the case of QD-GSH, Mag-GSH and Mag-Au-GSH, the formation of clusters and particle associations in solution could influence the DSL measurements. In any case, the four types of synthetized GSH-NPs show DLS diameters in the appropriate range for biomedical applications. DLS measurements over time show no important size differences after $48 \mathrm{~h}$ post-redispersion for any type of $\mathrm{NP}$, being the size increase lower than $1.2 \%$ in all cases.

\subsubsection{Z-Potential and Surface Characteristics}

To find out the electric charge surrounding NP surface we calculated the Z-potential. Figure 8a displays Z-potential versus $\mathrm{pH}$ for different NP colloidal solutions.

This figure indicates that all NP systems in this work show suitable features for biomedical applications, since the values in the Z-potential graphic reveals a relatively high colloidal stability in the studied $\mathrm{pH}$ range, and particularly at physiological $\mathrm{pH}$. 
These measurements shed information about the sign of the charge on the NP surface (as can be seen, all GSH samples present a negative Z-potential, which indicate an excess electric negative charge at the surface), that is also relevant for potential crosslinking applications. In general, the GSH molecule has thiol, amine and two carboxylic terminal groups available for linking to the NP surface. In the case of CdTe, due to the affinity between cadmium and sulfur, the GSH probably links the NP by its thiol group, keeping free the carboxylic terminal groups, responsible for the negative net charge. In order to study the linking mechanism for gold and iron oxide surfaces, we have prepared iron oxide and gold NPs, using a synthetic method similar to that used for Au-GSH and Mag-GSH NPs, but replacing the GSH with cysteamine (CYS), a molecule containing only two terminal groups: thiol and a primary amine. Table 2 shows Z-potential values at pH 7.2 for Au-GSH and Mag-GSH NPs, as well as for Mag-CYS and Au-CYS NPs. In a previous work [42], we demonstrated that the CYS molecule, links to the gold NP surface by the thiol group, due to the high affinity of thiols for the gold core, thus these CYS-capped NPs show positive Z-potential values due to the free amine terminal groups. However, in this work, we have obtained negative Z-potential for a Mag-CYS colloidal solution. This result points out that the affinity of amine terminal groups for the iron oxide core is higher than for the thiol group. Consequently, the GSH amine terminal group could have higher affinity for the iron oxide core than the GSH thiol group. We deduce that there are two different mechanisms for the linking between CYS and gold versus iron oxide NPs (Figure 8b,c). Interestingly, Mag-GSH NPs show the highest negative Z-potential values in all $\mathrm{pH}$ range (Figure 8a). This maximum negative charge could be better explained if we propose a different linking mechanism for this NP, in which GSH binds the NP surface by the amine group, with the thiol and the two carboxylic groups remaining free and, thus, contributing to the negative superficial charge. These results, which indicate that the iron oxide surface shows a higher affinity for amine than for thiol groups, can explain the higher negative Z-potential for the Mag-GSH NPs, due to the GSH linking to the iron oxide surface by its amine group instead of by the thiol group, contrary to what happens for gold NPs (Figure 8d,e).
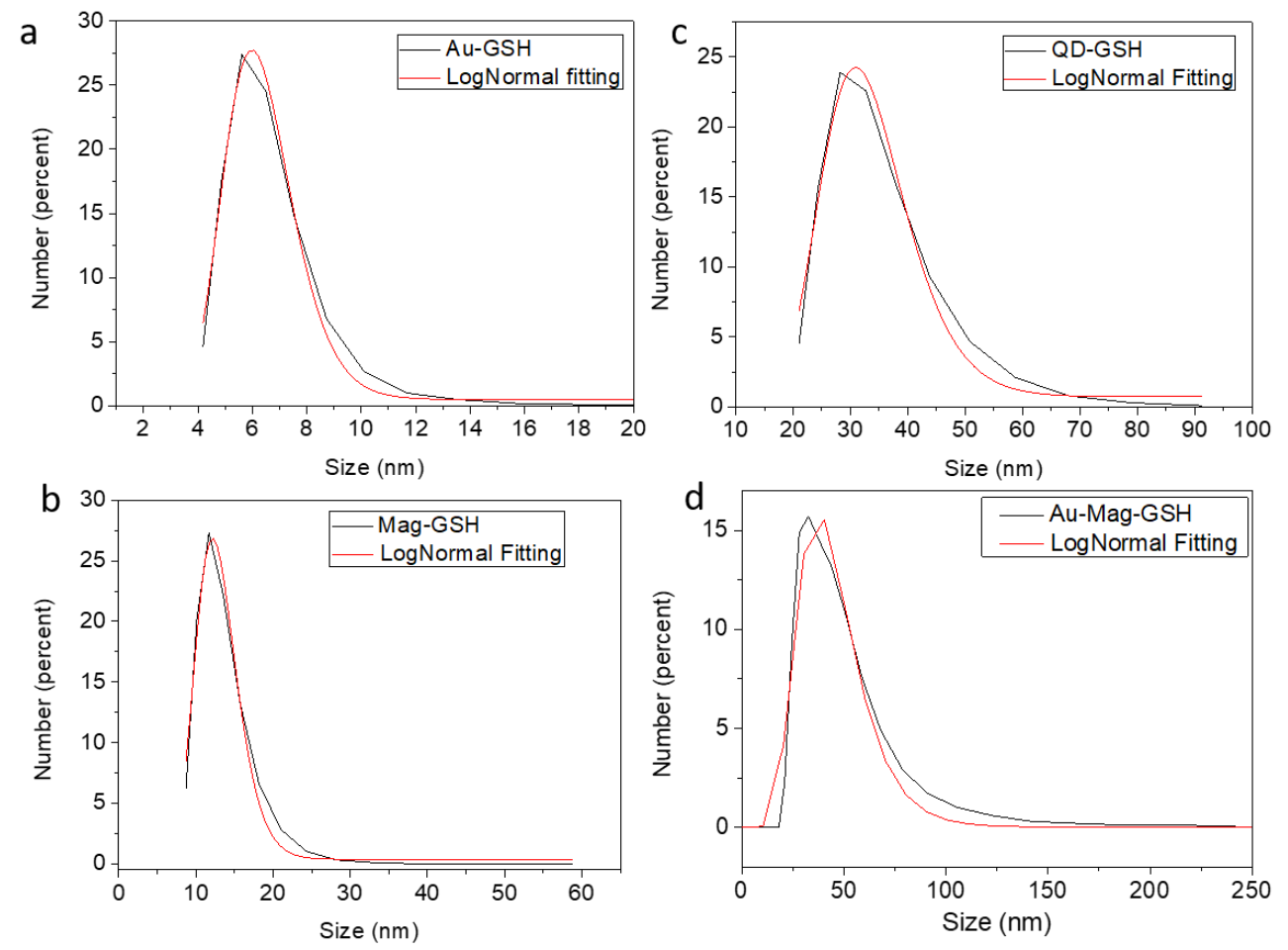

Figure 7. DLS size distributions of Au-GSH (a), Mag-GSH (b), CdTe-GSH1 (c) and Au-Mag-GSH (d) in PBS (phosphate-buffered saline) colloidal solutions. 


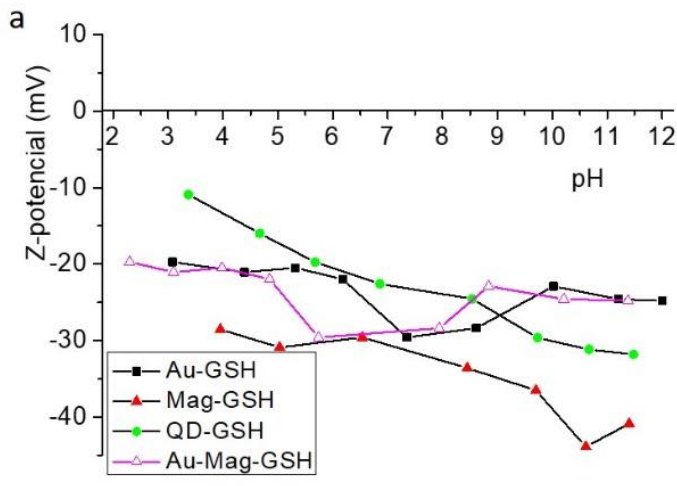

b
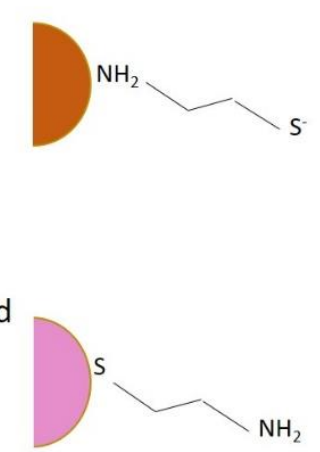

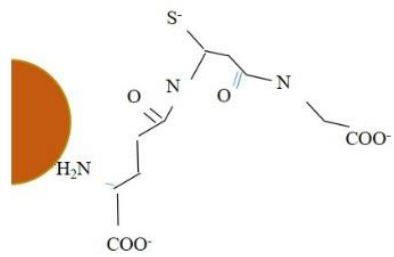

$\mathrm{e}$

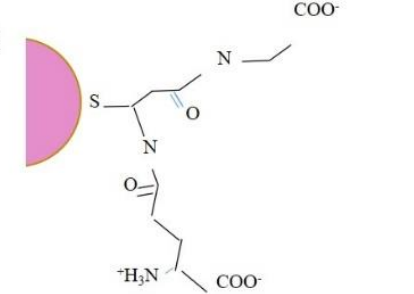

Figure 8. Z-potential versus $\mathrm{pH}$ for the different GSH capped NPs (a). Diagram of the cysteamine (CYS)-capped (b) and GSH-capped (c) magnetic-iron-oxide NPs. Diagram of the CYS-capped (d) and GSH-capped (e) gold NPs.

Table 2. Z-potential values obtained for gold and magnetic-iron-oxide NPs capped with CYS and with GSH at $25^{\circ} \mathrm{C}$ and in PBS solution.

\begin{tabular}{cc}
\hline Sample & Z-Potential (mV) \\
\hline Au-GSH & -32.8 \\
Mag-GSH & -33.5 \\
Au-CYS & +88.0 \\
Mag-CYS & -27.5 \\
\hline
\end{tabular}

In order to deepen knowledge of the differences in linking mechanisms between gold and iron oxide surfaces, we performed FTIR spectroscopy. Figure 9 shows the FTIR spectra for the free GSH ligand, as well as for Au-GSH, Mag-GSH and Au-Mag-GSH. The GSH spectrum present bands characteristics of amino $\left(3350 \mathrm{~cm}^{-1}\right)$, carboxylic $\left(1600 \mathrm{~cm}^{-1}\right)$ and thiol $\left(2530 \mathrm{~cm}^{-1}\right)$ groups. The peak at $2530 \mathrm{~cm}^{-1}$ that corresponds to the $\mathrm{S}-\mathrm{H}$ stretching vibration of GSH clearly disappeared in the Au-GSH and Au-Mag-GSH spectra, indicating that GSH anchors on the surface of these NPs through Au-S bonding. Conversely, a band at this position can be appreciated for Mag-GSH. The presence of the S-H stretching vibration band in this case, indicates a different GSH anchorage for this type of NP [52].

These FTIR spectra not only show a different GSH linking mechanism for gold and iron oxide NPs, but also suggests that gold is covering the iron oxide core in the case of hybrid iron oxide/gold NPs, as GSH linking occurs as it does for gold, with disappearance of the S-H stretching vibration of GSH.

These differences in the linking mechanism could also introduce differences in the shell thickness, as we have previously commented. A schematic representation of this linking mechanism is shown in Figure 8 b through Figure 8e.

\subsubsection{Temperature Effect}

NPs used for biological applications require appropriate colloidal stability at human body temperature, ranging from 37 to $40^{\circ} \mathrm{C}$, as well as at different saline concentrations. We studied the stability of the NP dispersions under different environmental conditions (temperature and salinity). DLS experiments were also performed at 37 and $40^{\circ} \mathrm{C}$; and also subjecting NPs to an environment of a higher ionic strength, after dispersion of NPs in a $0.18 \mathrm{M}$ solution of $\mathrm{NaCl}$. Table 3 shows NP diameters obtained by DLS for temperatures of 25,37 and $40{ }^{\circ} \mathrm{C}$ for all NPs in PBS dispersions, as well as at $25^{\circ} \mathrm{C}$ in a $0.18 \mathrm{M} \mathrm{NaCl}$ dispersion. Results reveal that the only NPs that present a colloidal stability dependence with temperature is Au-GSH, which has an extremely low hydrodynamic size at $25{ }^{\circ} \mathrm{C}$. 
The rest of the NPs do not show a strong variation in the dynamic size when temperature increases. However, when the ionic strength changes, a significant increase in the dynamic diameter is observed for all GSH-NPs. In all cases NPs remain in the appropriate range for biomedical applications.

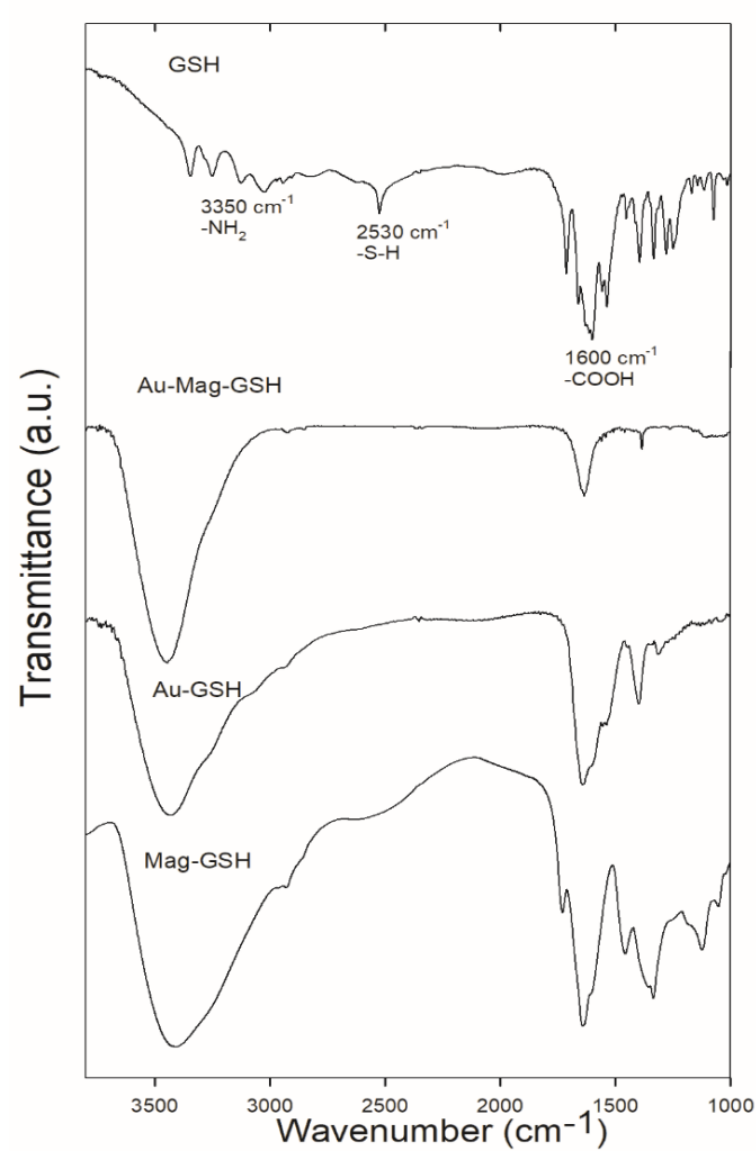

Figure 9. Fourier transform infrared (FTIR) spectra for free GSH ligand as well as for Au-Mag-GSH, $\mathrm{Au}-\mathrm{GSH}$ and Mag-GSH NPs.

Table 3. NPs and their corresponding dynamic light scattering (DLS) hydrodynamic sizes at different temperatures.

\begin{tabular}{cccc}
\hline Sample & Temperature $\left({ }^{\circ} \mathbf{C}\right)$ & Dispersion Medium & DLSAverage Size (nm) \\
\hline \multirow{3}{*}{ Au-GSH } & 25 & PBS & $6.2 \pm 1.2$ \\
& 37 & PBS & $24.9 \pm 2.2$ \\
& 40 & PBS & $13.5 \pm 1.5$ \\
& 25 & $0.18 \mathrm{NaCl}$ & $28.2 \pm 2.5$ \\
\hline \multirow{2}{*}{ QD-GSH } & 25 & PBS & $32.6 \pm 1.3$ \\
& 37 & PBS & $39.3 \pm 2.7$ \\
& 40 & PBS & $36.3 \pm 2.7$ \\
& 25 & $0.18 \mathrm{NaCl}$ & $58.1 \pm 2.5$ \\
\hline \multirow{2}{*}{ Mag-GSH } & 25 & PBS & $12.7 \pm 1.2$ \\
& 37 & PBS & $11.3 \pm 1.6$ \\
& 40 & PBS & $12.9 \pm 1.7$ \\
& 25 & $0.18 \mathrm{NaCl}$ & $29.2 \pm 2.6$ \\
\hline
\end{tabular}

All these colloidal characterization results confirm that the NP core is capped by GSH molecules that properly functionalize the NP, allowing for subsequent biofunctionalization and thus corroborating previously presented (S)TEM results. Functionalized NPs show 
colloidal stability at physiological $\mathrm{pH}$ and temperature values. Indeed, GSH-functionalized NPs show a higher colloidal stability at physiological $\mathrm{pH}$ and temperatures than citratecapped gold NPs [42].

\subsection{Cell Viability and Cytotoxicity}

As previously commented, one key property of NPs to consider for medical applications is cytotoxicity, and thus we performed MTT cell viability assays for Jurkat cells incubated with the synthesized NPs. In Figure 10, viability for cells cultured in the presence of NPs at $1.5 \mu \mathrm{g} / \mathrm{mL}$ (a) or $15 \mu \mathrm{g} / \mathrm{mL}$ (10.b) is shown. These graphics show mean values and standard errors for the percentage of viable cells when cultured in the presence of AuGSH NPs (A), Mag-GSH (B), Au-Mag-GSH NPs (C) or QD-GSH NPs (D) considering 100\% the viability of cells cultured in the absence of NPs (NP(-)) as a control. Each experiment was performed independently in triplicate.

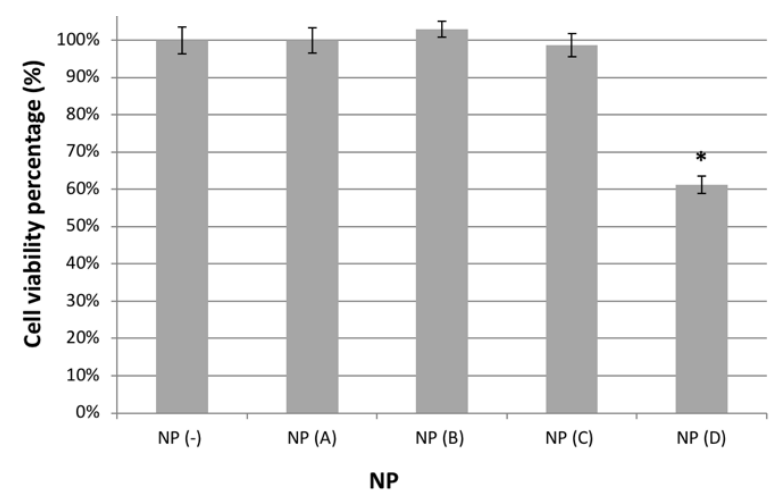

(a)

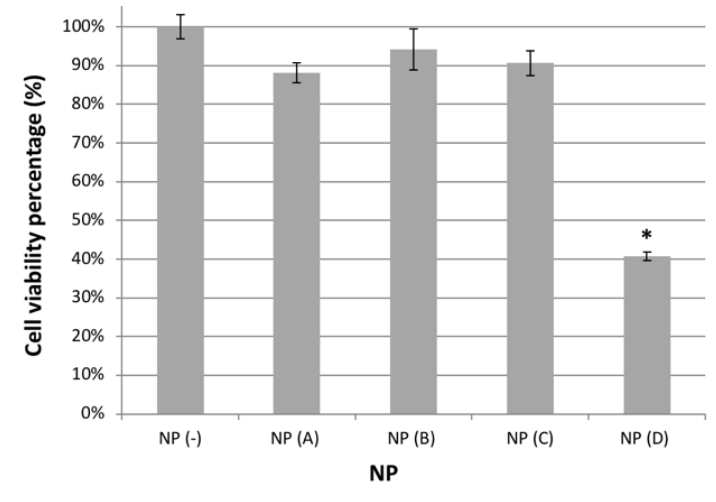

(b)

Figure 10. MTT cell viability assay for Jurkat tumor cells incubated with the indicated type of NP at $1.5 \mu \mathrm{g} / \mathrm{mL}$ (a) or $15 \mu \mathrm{g} / \mathrm{mL}$ (b). Percentage of viable cells as compared to cells cultured in the absence of NP (considered as $100 \%$ viability) is shown. Mean and standard error are shown for each sample. A representative experiment out of three is shown. Statistically significant differences $(p<0.05)$ are marked with a star $(*)$.

As shown in Figure 10a, all NPs, except for QD-GSH, are not toxic at concentrations usually utilized in the literature $(1.5 \mu \mathrm{g} / \mathrm{mL})$ with viabilities similar to those observed in control cultures in the absence of NPs, indicating the extremely low cytotoxicity of these NPs. Even at concentrations 10 times higher $(15 \mu \mathrm{g} / \mathrm{mL})$, these NPs have only a marginal effect on cell viability (90\% viability) (Figure 10b). At this concentration $(15 \mu \mathrm{g} / \mathrm{mL}$ ), cell viability is $88 \%$ for Au-GSH, $94 \%$ for Mag-GSH and between $88 \%$ and $94 \%$ for the hybrid Au-Mag-GSH, indicating that their toxicity profile is very favorable for in vivo applications, with Mag-GSH being the NP with the best toxicity profile (Figure 10b).

On the other hand QD-GSH NPs, have an effect on cell viability with percentages of $61 \%$ and $41 \%$ for NP concentrations of 1.5 and $15 \mu \mathrm{g} / \mathrm{mL}$, respectively.

To better ascertaining the toxicity profile, we performed a dose respond experiment with NP concentrations up to $960 \mu \mathrm{g} / \mathrm{mL}$. As shown in Figure 11, Mag-GSH and Au-MagGSH have a very favorable toxicity profile, while QD-GSH are more toxic, with Au-GSH having an intermediate toxicity.

The lower viability of cells that were cultured in the presence of QD-GSH is due to the inherent toxicity of the CdTe material, that prevails even when capped with GSH. Therefore, until an alternative capping, able to seal off the CdTe material, is engineered, this type of NP should be utilized as a biomarker only for in vitro experiments, but not for in vivo applications. Interestingly, hybrid Au-Mag-GSH NPs show better toxicity profile than Au-GSH NPs, which can be due to the small size of this gold NPs [53]. 


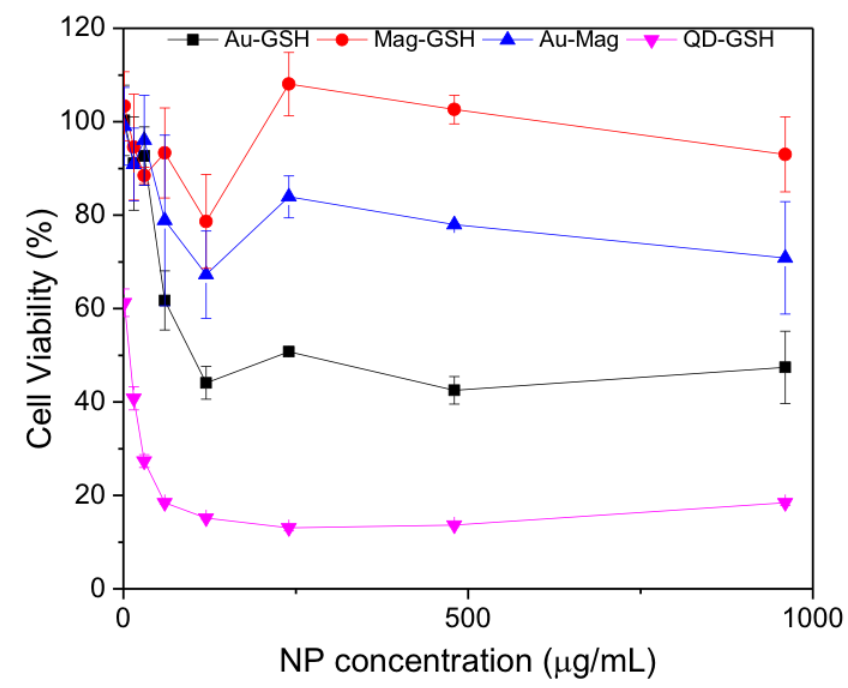

Figure 11. MTT cell viability assay for Jurkat tumor cells incubated with the indicated NP at 1.5; 15; $30 ; 60 ; 120 ; 240 ; 480$ and $960 \mu \mathrm{g} / \mathrm{mL}$. Percentage of viable cells, as compared to cells cultured in the absence of NP (considered as 100\% viability), is shown. Mean values and standard error for three experiments are shown. Viability of cells cultured in the presence of $10 \%$ dimethyl sulfoxide (DMSO) is $8.79 \%$.

\section{Discussion}

In this work we used glutathione as a capping agent to functionalize gold/iron oxide NPs for biomedical applications, prepared by a novel and simple route. We also compared the results obtained for this GSH-gold/magnetic iron oxide NPs with those obtained from other GSH capped NPs. First, we confirmed that the functionalization preserves the structure and physical properties that confer each of these NPs with their usefulness for biomedical applications. The work was focused on determining GSH-capping effectiveness, linkage characteristics between GSH and NP surfaces and the influence of GSH capping on the physical properties and cytotoxicity of NPs.

We described a route to obtain Au-Mag-GSH, a hybrid NP encompassing the biomedical performances of both, magnetic iron oxide and gold. Although different authors have proposed different routes to prepare hydrophilic synthesizes [54,55], we have designed a simple route to obtain, in two simple steps, hydrophilic functionalized magnetic iron oxide-gold NPs. The formation of hybrid NPs in which both phases, iron oxide and gold, are present in the same NP was confirmed by results obtained from TEM and UV-visible absorption. This last technique allowed us to identify the characteristic gold LRSP band, but with a clear red shift when compared to the Au-GSH LSPR band, which according to Bhatia et al. [56] can be attributed to the interfacial charge transfer between gold and magnetic-iron-oxide. TEM results revealed formation of spherical and homogeneous GSHNPs of $7 \mathrm{~nm}$ average size with a core formed by gold and either iron or an iron compound. EDX results also revealed the existence of both, iron and gold in the same NP. Considering that our first synthesis step was the formation of an iron oxide core, it is reasonable to suggest formation of a gold NP shell surrounding the magnetic-iron-oxide. In any case, since no region of pure gold was found via EDX maps for iron-gold NPs (not shown here), but areas with very close diameters for the spectral signals of $\mathrm{Au}$ and Fe, if the gold was forming a shell, it would be assumed to be thin, although at least a few monolayers-thick since the gold structure was detected by the beam. Thus, the nature of this NP, whether it is formed by an iron compound or magnetite, or even by an alloy of gold and iron could not have been concluded by the applied (S)TEM techniques. In any case, both the red-shift in the LSPR absorption band of these NPs and the sequence followed during the synthesis method employed in the study (iron oxide nucleation followed by gold addition) seemed to indicate that a core-shell structure should have been formed. Moreover the fact that the 
FTIR results showed a linkage mechanism for GSH to gold/iron oxide akin to that seen for gold NPs, further strengthened the hypothesis that a core iron oxide, that confers magnetic properties, is surrounded by gold that justifies the linking mechanism.

We have compared results obtained for these hybrid NPs with those obtained for pure gold, magnetic-iron-oxide and CdTe, also capped with GSH. In the case of QDs (CdTe) and according to recent work [57], extremely small NPs immersed in GSH capping appeared. This was probably due to the excess of GSH added during the synthesis and to the high affinity of the GSH thiol group for the CdTe surface. However, the process allowed for size control, providing a tunable photoluminescence [14].

GSH capping contributes to the formation of spherical and homogeneous NPs in the case of iron oxide and gold NPs. Magnetic NPs (Mag-GSH) of $6 \mathrm{~nm}$ average size are obtained, while smaller, and less aggregated NPs are formed in the case of pure gold NPs (Au-GSH). For the latter, the presence of gold was confirmed by UV-visible absorption and (S)TEM. These NPs show a PL emission in the blue range that, in agreement with our previous studies, can be explained considering the small size of gold cores and the ligand-metal charge transfer transition due to the thiol group of the GSH capping. This characteristic could be an interesting feature, making these NPs suitable not only for diagnostics, therapy or further bioconjugations but also for direct biomarker applications [42].

We used the HRTEM technique to prove the homogeneous composition, spherical shape and size (under $10 \mathrm{~nm}$ ) of NPs, and also to visualize the GSH layer. In order to prove that this layer was composed of GSH, the sulfur present in the glutathione was followed by energy-dispersive X-ray spectroscopy. In this way, the presence of such element, detected at the NP surface, confirms that the amorphous layer at the NP surface is the GSH capping. Additionally, results obtained from Z-potential measurements allowed us to deepen the GSH linkage mechanism. The GSH molecule contains different terminal functional groups: thiol, amine and two carboxylic groups. Knowing the group that molecules use to link to the NP surface and consequently the remaining free moieties, is crucial for the successful design of subsequent crosslinking processes. With this goal, we used a simple molecule, cysteamine, to functionalize gold and iron oxide NPs. Our results, which yielded negative Z-potential values for gold NPs functionalized with cysteamine, confirmed the high affinity between thiol and gold surface. On the other hand, the positive Z-potential obtained for cysteamine capped iron oxide, revealed a higher affinity between the iron oxide surface and amine than between this iron oxide and the thiol group. This suggests that GSH does not use the thiol group to link to iron oxide GSH-NPs as it does for $\mathrm{Au}$ containing NPs. Whereas Au-GSH and Au-Mag-GSH lose the characteristic S-H stretching vibration peak, this band was observed for Mag-GSH, indicating that in this last case the thiol group was not used to link the NP surface. This is in agreement with recent works that propose a conjugation of GSH capped iron oxide NPs by the free thiol group [58]. Several authors propose the iron oxide functionalization by carboxylic groups [59]. However, the relatively high negative Z-potential values obtained for Mag-GSH in all the studied $\mathrm{pH}$ range together with our cysteamine-iron oxide results led us to propose the linkage by the amine group for Mag-GSH. NPs using gold (Au-GSH and Au-Mag-GSH) were clearly linked by the thiol group.

Concerning colloidal stability, DLS experiments reveal hydrodynamic sizes bigger than those obtained by HRTEM (NP core plus GSH layer). In the case of Au-GSH, this difference could be explained by the presence of extra hydrate layers in aqueous medium. However, for all other NPs differences between DLS and TEM diameters were significantly bigger. For Mag-GSH and Au-Mag-GSH the magnetic character of NPs originated in magnetic interactions between particles, especially for Mag-GSH, in which the observed aggregation was higher. It is interesting to remark that DLS average sizes were inversely proportional to the estimated GSH layer thickness. A wider GSH layer provided less aggregation in aqueous colloidal solution. In the case of QD-GSH, as we observed by TEM, NPs were not well dispersed, and the cores were immersed in the GSH capping. This could be the 
reason for the relatively high DLS size $(32.6 \mathrm{~nm})$ for non-magnetic NPs with a core size under $3 \mathrm{~nm}$.

Nevertheless, at the studied conditions, all NPs showed DLS diameters smaller than $50 \mathrm{~nm}$, and colloidal stability in an appropriate range, although further work is in process to check colloidal stability under different conditions to perform additional steps of multimodal crosslinking.

Finally, we showed how GSH functionalization was also able to confer biocompatibility to most of the studied NPs. Au-GSH, Mag-GSH and Au-Mag-GSH show cell viability percentages similar to controls at concentrations up to $30 \mu \mathrm{g} / \mathrm{mL}$; with Au-GSH and MagGSH, maintaining the low toxicity profile up to concentrations of $960 \mu \mathrm{g} / \mathrm{mL}$. Interestingly enough, hybrid Au-Mag-GSH NPs showed a better toxicity profile than Au-GSH NPs.

However, this extremely low cytotoxicity was not observed for QD-GSH particles, in which the GSH capping cannot passivate cadmium inherent toxicity. Different authors propose different mechanisms for CdTe toxicity, such as photo oxidation and the consequent formation of degradation products that generate superoxide anions, which might lead to rust and corrosion of NP surfaces [60]. Concomitantly, in the case of QDs made of cadmium, cytotoxicity is a consequence of the release of free cadmium ions $\left(\mathrm{Cd}^{2+}\right)$ that are highly toxic. However, QD-GSH NPs can still be used as biomarkers for in vitro experiments.

\section{Conclusions}

We have designed a new and simple synthetic method to prepare hydrophilic metallic NPs capped with GSH (including, gold/iron oxide hybrid NPs). We have studied the structural, morphological and colloidal properties, as well as the cytotoxicity, for these new hybrid NPs and for iron-containing NPs, gold NPs and fluorescent NPs, previously prepared for us and also covered with GSH. We have obtained, in all cases, spherical highly crystalline and homogeneous NPs with sizes under $10 \mathrm{~nm}$. The combination of TEM and STEM techniques also allowed visualization of the amorphous GSH layer, confirming an adequate functionalization. All GSH-capped NPs showed colloidal stability, after 48-h post-redispersion, especially at the physiological $\mathrm{pH}$ range. This colloidal stability did not significantly decrease by increasing temperature up to $40^{\circ} \mathrm{C}$. Colloidal characterization allowed us to determine the moiety in GSH that binds to the NP surface. We have deduced that GSH links iron-containing NP surface by its amine group and not by the thiol groups, as is the case for the rest of studied NPs. This knowledge is crucial to determine the free terminal groups in the GSH capping, which is pivotal for the design of subsequent crosslinking strategies. The developed NPs show high biocompatibility with low cytotoxicity even at high concentrations, with the exception of QD-GSH, that could be only suitable for in vitro biomedical applications.

Author Contributions: Conceptualization: R.L., O.B.-M. and F.G.-C.; Nanoparticle Synthesis: C.F.-P., J.B.-L. and R.L.; Magnetic experiments: J.B.-L.; Optical measurements: R.L. and R.F.-C.; DLS experiments: E.F. and O.B.-M.; FTIR experiments: M.P.Y. Electron microscopy: J.M.M., A.M.B., F.M.M. and A.J.S., Cell viability and cytotoxicity: C.F.-P., J.P.M.-M., R.F.-C. and F.G.-C.; Data curation: All author; Writing—original draft preparation: R.L., J.M.M., C.F.P. and F.G.-C.; writing—review and editing: All authors; Project administration: R.L.; Funding acquisition: F.G.-C. and O.B.-M. All authors have read and agreed to the published version of the manuscript.

Funding: This work was supported by Iniciativa Territorial Integrada, Junta de Andalucia (PI-00302017) and by the Ministry of Education and Science, Instituto Salud Carlos III (PI16-00784) fondo covid-19 COV20/00173) for F.G-C and by the Spanish Ministry of Economy and Competitiveness Program "Plan I+D+i", subprogram "Retos" (MAT2015-67354-R) for O.B-M.

Institutional Review Board Statement: Not applicable.

Informed Consent Statement: Not applicable.

Data Availability Statement: Not applicable. 
Acknowledgments: The authors would like to thank the Core Biomedical Research (sc-IBM) Facility and the Core Science and Technology Research Facility (sc-ICyT) of University Cadiz for the use of core infrastructure, as well as to the microscopy facilities of CITIUS-Universidad de Sevilla (Spain).

Conflicts of Interest: The authors declare no conflict of interest.

\section{References}

1. Bakhtiari-Asl, F.; Divband, B.; Mesbahi, A.; Gharehaghaji, N. Bimodal magnetic resonance imaging-computed tomography nanoprobes: A Review. Nanomed. J. 2020, 7, 1-12. [CrossRef]

2. Gharpure, S.; Akash, A.; Ankamwar, B. A Review on Antimicrobial Properties of Metal Nanoparticles. J. Nanosci. Nanotechnol. 2020, 20, 3303-3339. [CrossRef] [PubMed]

3. Giljohann, D.A.; Mirkin, C.A. Drivers of biodiagnostic development. Nature 2009, 462, 461-464. [CrossRef] [PubMed]

4. Wang, J.; Li, P.; Yu, Y.; Fu, Y.; Jiang, H.; Lu, M.; Sun, Z.; Jiang, S.; Lu, L.; Wu, M.X. Pulmonary surfactant-biomimetic nanoparticles potentiate heterosubtypic influenza immunity. Science 2020, 367, 869. [CrossRef]

5. Lu, L.; Qi, S.; Chen, Y.; Luo, H.; Huang, S.; Yu, X.; Luo, Q.; Zhang, Z. Targeted immunomodulation of inflammatory monocytes across the blood-brain barrier by curcumin-loaded nanoparticles delays the progression of experimental autoimmune encephalomyelitis. Biomaterials 2020, 245, 119987. [CrossRef]

6. Mirzaei, M.; Akbari, M.E.; Mohagheghi, M.A.; Ziaee, S.A.M.; Mohseni, M. A novel biocompatible nanoprobe based on lipoproteins for breast cancer cell imaging. Nanomed. J. 2020, 7, 73-79. [CrossRef]

7. Shi, S.; Vissapragada, R.; Abi Jaoude, J.; Huang, C.; Mittal, A.; Liu, E.; Zhong, J.; Kumar, V. Evolving role of biomaterials in diagnostic and therapeutic radiation oncology. Bioact. Mater. 2020, 5, 233-240. [CrossRef]

8. Van Elk, M.; Murphy, B.P.; Eufrasio-da-Silva, T.; O’Reilly, D.P.; Vermonden, T.; Hennink, W.E.; Duffy, G.P.; Ruiz-Hernandez, E. Nanomedicines for advanced cancer treatments: Transitioning towards responsive systems. Int. J. Pharm. 2016, 515, 132-164. [CrossRef]

9. Chen, D.; Zhao, C.; Ye, J.; Li, Q.; Liu, X.; Su, M.; Jiang, H.; Amatore, C.; Selke, M.; Wang, X. In Situ Biosynthesis of Fluorescent Platinum Nanoclusters: Toward Self-Bioimaging-Guided Cancer Theranostics. Acs Appl. Mater. Interfaces 2015, 7, 18163-18169. [CrossRef] [PubMed]

10. Rehman, F.U.; Du, T.; Shaikh, S.; Jiang, X.; Chen, Y.; Li, X.; Yi, H.; Hui, J.; Chen, B.; Selke, M.; et al. Nano in nano: Biosynthesized gold and iron nanoclusters cargo neoplastic exosomes for cancer status biomarking. Nanomed. Nanotechnol. Biol. Med. 2018, 14, 2619-2631. [CrossRef] [PubMed]

11. Meng, W.; He, C.; Hao, Y.; Wang, L.; Li, L.; Zhu, G. Prospects and challenges of extracellular vesicle-based drug delivery system: Considering cell source. Drug Deliv. 2020, 27, 585-598. [CrossRef]

12. He, Z.; Jiang, R.; Long, W.; Huang, H.; Liu, M.; Feng, Y.; Zhou, N.; Ouyang, H.; Zhang, X.; Wei, Y. Red aggregation-induced emission luminogen and Gd3+ codoped mesoporous silica nanoparticles as dual-mode probes for fluorescent and magnetic resonance imaging. J. Colloid Interface Sci. 2020, 567, 136-144. [CrossRef]

13. Larson, D.R.; Zipfel, W.R.; Williams, R.M.; Clark, S.W.; Bruchez, M.P.; Wise, F.W.; Webb, W.W. Water-soluble quantum dots for multiphoton fluorescence imaging in vivo. Science 2003, 300, 1434-1436. [CrossRef]

14. Beato-Lopez, J.J.; Espinazo, M.L.; Fernandez-Ponce, C.; Blanco, E.; Ramirez-del-Solar, M.; Dominguez, M.; Garcia-Cozar, E.; Litran, R. CdTe quantum dots linked to Glutathione as a bridge for protein crosslinking. J. Lumin. 2017, 187, 193-200. [CrossRef]

15. Qu, Z.; Liu, L.; Sun, T.; Hou, J.; Sun, Y.; Yu, M.; Diao, Y.; Lu, S.; Zhao, W.; Wang, L. Synthesis of bifunctional carbon quantum dots for bioimaging and anti-inflammation. Nanotechnology 2020, 31, 175102. [CrossRef] [PubMed]

16. Kayili, H.M.; Salih, B. Fast and efficient proteolysis by reusable pepsin-encapsulated magnetic sol-gel material for mass spectrometry-based proteomics applications. Talanta 2016, 155, 78-86. [CrossRef]

17. Jose, J.; Kumar, R.; Harilal, S.; Mathew, G.E.; Parambi, D.G.T.; Prabhu, A.; Uddin, M.S.; Aleya, L.; Kim, H.; Mathew, B. Magnetic nanoparticles for hyperthermia in cancer treatment: An emerging tool. Environ. Sci. Pollut. Res. 2019. [CrossRef]

18. Sharma, S.K.; Shrivastava, N.; Rossi, F.; Le Duc, T.; Nguyen Thi Kim, T. Nanoparticles-based magnetic and photo induced hyperthermia for cancer treatment. Nano Today 2019, 29. [CrossRef]

19. Karimzadeh, I.; Dizaji, H.R.; Aghazadeh, M. Development of a facile and effective electrochemical strategy for preparation of iron oxides ( $\mathrm{Fe} 3 \mathrm{O} 4$ and gamma-Fe2O3) nanoparticles from aqueous and ethanol mediums and in situ PVC coating of Fe3O4 superparamagnetic nanoparticles for biomedical applications. J. Magn. Magn. Mater. 2016, 416, 81-88. [CrossRef]

20. Bomati-Miguel, O.; Lahoz, R.; Lennikov, V.; Naghilou, A.; Subotic, A.; Angel Rodriguez, M.; Rentenberger, C.; Kautek, W.; IEEE. Liquid-Assisted Pulsed Laser Ablation: A Novel Route to Produce Multifunctional Contrast Agents for Multimodal Imaging Diagnosis. In Proceedings of the European Conference on Lasers and Electro-Optics and European Quantum Electronic Conference, Munich, Germany, 23-27 June 2017.

21. Du, Y.; Liu, X.; Liang, Q.; Liang, X.-J.; Tian, J. Optimization and Design of Magnetic Ferrite Nanoparticles with Uniform Tumor Distribution for Highly Sensitive MRI/MPI Performance and Improved Magnetic Hyperthermia Therapy. Nano Lett. 2019, 19, 3618-3626. [CrossRef]

22. Soares, P.I.P.; Laia, C.A.T.; Carvalho, A.; Pereira, L.C.J.; Coutinho, J.T.; Ferreira, I.M.M.; Novo, C.M.M.; Borges, J.P. Iron oxide nanoparticles stabilized with a bilayer of oleic acid for magnetic hyperthermia and MRI applications. Appl. Surf. Sci. 2016, 383, 240-247. [CrossRef] 
23. Scialabba, C.; Puleio, R.; Peddis, D.; Varvaro, G.; Calandra, P.; Cassata, G.; Cicero, L.; Licciardi, M.; Giammona, G. Folate targeted coated SPIONs as efficient tool for MRI. Nano Res. 2017, 10, 3212-3227. [CrossRef]

24. Neuwelt, E.A.; Varallyay, C.G.; Manninger, S.; Solymosi, D.; Haluska, M.; Hunt, M.A.; Nesbit, G.; Stevens, A.; Jerosch-Herold, M.; Jacobs, P.M.; et al. The potential of ferumoxytol nanoparticle magnetic resonance imaging, perfusion, and angiograpgy in central nervous system malignancy: A pilot study. Neurosurgery 2007, 60, 601-611. [CrossRef]

25. Bai, X.; Wang, Y.; Song, Z.; Feng, Y.; Chen, Y.; Zhang, D.; Feng, L. The Basic Properties of Gold Nanoparticles and their Applications in Tumor Diagnosis and Treatment. Int. J. Mol. Sci. 2020, 21, 2480. [CrossRef]

26. Sugumaran, S.; Jamlos, M.F.; Ahmad, M.N.; Bellan, C.S.; Schreurs, D. Nanostructured materials with plasmonic nanobiosensors for early cancer detection: A past and future prospect. Biosens. Bioelectron. 2018, 100, 361-373. [CrossRef] [PubMed]

27. Ru, F.; Du, P.; Lu, X. Efficient ratiometric fluorescence probe utilizing silicon particles/gold nanoclusters nanohybrid for "onoff-on" bifunctional detection and cellular imaging of mercury (II) ions and cysteine. Anal. Chim. Acta 2020, 1105, 139-146. [CrossRef] [PubMed]

28. Patel, P.C.; Giljohann, D.A.; Daniel, W.L.; Zheng, D.; Prigodich, A.E.; Mirkin, C.A. Scavenger Receptors Mediate Cellular Uptake of Polyvalent Oligonucleotide-Functionalized Gold Nanoparticles. Bioconjug. Chem. 2010, 21, 2250-2256. [CrossRef]

29. Rawson, S.D.; Maksimcuka, J.; Withers, P.J.; Cartmell, S.H. X-ray computed tomography in life sciences. BMC Biol. 2020, 18, 21. [CrossRef]

30. Chen, M.; Tang, S.; Guo, Z.; Wang, X.; Mo, S.; Huang, X.; Liu, G.; Zheng, N. Core-Shell Pd@Au Nanoplates as Theranostic Agents for In-Vivo Photoacoustic Imaging, CT Imaging, and Photothermal Therapy. Adv. Mater. 2014, 26, 8210-8216. [CrossRef] [PubMed]

31. Lewinski, N.; Colvin, V.; Drezek, R. Cytotoxicity of nanoparticles. Small 2008, 4, 26-49. [CrossRef]

32. Hu, M.; Petrova, H.; Sekkinen, A.R.; Chen, J.; McLellan, J.M.; Li, Z.-Y.; Marquez, M.; Li, X.; Xia, Y.; Hartland, G.V. Optical properties of Au-Ag nanoboxes studied by single nanoparticle spectroscopy. J. Phys. Chem. B 2006, 110, 19923-19928. [CrossRef] [PubMed]

33. Piergies, N.; Ocwieja, M.; Paluszkiewicz, C.; Kwiatek, W.M. Nanoparticle stabilizer as a determining factor of the drug/gold surface interaction: SERS and AFM-SEIRA studies. Appl. Surf. Sci. 2021, 537, 147897. [CrossRef]

34. Takemura, K.; Lee, J.; Suzuki, T.; Hara, T.; Abe, F.; Park, E.Y. Ultrasensitive detection of norovirus using a magnetofluoroimmunoassay based on synergic properties of gold/magnetic nanoparticle hybrid nanocomposites and quantum dots. Sens. Actuators B-Chem. 2019, 296, 147897. [CrossRef]

35. Carril, M.; Fernandez, I.; Rodriguez, J.; Garcia, I.; Penades, S. Gold-Coated Iron Oxide Glyconanoparticles for MRI, CT, and US Multimodal Imaging. Part. Part. Syst. Charact. 2014, 31, 81-87. [CrossRef]

36. Lv, W.; Shen, Y.; Yang, H.; Yang, R.; Cai, W.; Zhang, J.; Yuan, L.; Duan, Y.; Zhang, L. A Novel Bimodal Imaging Agent Targeting HER2 Molecule of Breast Cancer. J. Immunol. Res. 2018, 2018, 6202876. [CrossRef]

37. Albanese, A.; Tang, P.S.; Chan, W.C.W. The Effect of Nanoparticle Size, Shape, and Surface Chemistry on Biological Systems. In Annual Review of Biomedical Engineering; Yarmush, M.L., Ed.; University of Toronto: Toronto, ON, Canada, 2012; Volume 14, pp. 1-16.

38. Uzun, O.; Hu, Y.; Verma, A.; Chen, S.; Centrone, A.; Stellacci, F. Water-soluble amphiphilic gold nanoparticles with structured ligand shells. Chem. Commun. 2008, 2, 196-198. [CrossRef]

39. Verma, A.; Stellacci, F. Effect of Surface Properties on Nanoparticle-Cell Interactions. Small 2010, 6, 12-21. [CrossRef]

40. Verma, A.; Uzun, O.; Hu, Y.; Hu, Y.; Han, H.-S.; Watson, N.; Chen, S.; Irvine, D.J.; Stellacci, F. Surface-structure-regulated cell-membrane penetration by monolayer-protected nanoparticles (vol 7, pg 588, 2008). Nat. Mater. 2013, 12, 376. [CrossRef]

41. Beato-Lopez, J.J.; Fernandez-Ponce, C.; Blanco, E.; Barrera-Solano, C.; Ramirez-del-Solar, M.; Dominguez, M.; Garcia-Cozar, F.; Litran, R. Preparation and Characterization of Fluorescent CdS Quantum Dots used for the Direct Detection of GST Fusion Proteins Regular Paper. Nanomater. Nanotechnol. 2012, 2, 2012. [CrossRef]

42. Fernandez-Ponce, C.; Munoz-Miranda, J.P.; de los Santos, D.M.; Aguado, E.; Garcia-Cozar, F.; Litran, R. Influence of size and surface capping on photoluminescence and cytotoxicity of gold nanoparticles. J. Nanopart. Res. 2018, 20, 305. [CrossRef]

43. Simpson, C.A.; Salleng, K.J.; Cliffel, D.E.; Feldheim, D.L. In vivo toxicity, biodistribution, and clearance of glutathione-coated gold nanoparticles. Nanomed. Nanotechnol. Biol. Med. 2013, 9, 257-263. [CrossRef]

44. Chen, G.; Zhang, Y.; Peng, Z.; Huang, D.; Li, C.; Wang, Q. Glutathione-capped quantum dots for plasma membrane labeling and membrane potential imaging. Nano Res. 2019, 12, 1321-1326. [CrossRef]

45. Beltran, A.M.; Manuel, J.M.; Litran, R.; Felix, E.; Santos, A.J.; Morales, F.M.; Bomati-Miguel, O. (S)TEM structural and compositional nanoanalyses of chemically synthesized glutathione-shelled nanoparticles. Appl. Nanosci. 2020, 10, 2295-2301. [CrossRef]

46. Crespo, P.; Litran, R.; Rojas, T.C.; Multigner, M.; de la Fuente, J.M.; Sanchez-Lopez, J.C.; Garcia, M.A.; Hernando, A.; Penades, S.; Fernandez, A. Permanent magnetism, magnetic anisotropy, and hysteresis of thiol-capped gold nanoparticles. Phys. Rev. Lett. 2004, 93, 087204. [CrossRef] [PubMed]

47. Beato-Lopez, J.J.; Dominguez, M.; Ramirez-del-Solar, M.; Litran, R. Glutathione-magnetite nanoparticles: Synthesis and physical characterization for application as MRI contrast agent. Sn Appl. Sci. 2020, 2, 1202. [CrossRef]

48. Umut, E.; Pineider, F.; Arosio, P.; Sangregorio, C.; Corti, M.; Tabak, F.; Lascialfari, A.; Ghigna, P. Magnetic, optical and relaxometric properties of organically coated gold-magnetite ( $\mathrm{Au}-\mathrm{Fe} 3 \mathrm{O} 4)$ hybrid nanoparticles for potential use in biomedical applications. J. Magn. Magn. Mater. 2012, 324, 2373-2379. [CrossRef]

49. Himstedt, R.; Rusch, P.; Hinrichs, D.; Kodanek, T.; Lauth, J.; Kinge, S.; Siebbeles, L.D.A.; Dorfs, D. Localized Surface Plasmon Resonances of Various Nickel Sulfide Nanostructures and Au-Ni3S2 Core-Shell Nanoparticles. Chem. Mater. 2017, 29, 7371-7377. [CrossRef] 
50. Paramasivam, G.; Kayambu, N.; Rabel, A.M.; Sundramoorthy, A.K.; Sundaramurthy, A. Anisotropic noble metal nanoparticles: Synthesis, surface functionalization and applications in biosensing, bioimaging, drug delivery and theranostics. Acta Biomater. 2017, 49, 45-65. [CrossRef]

51. Bernal, S.; Botana, F.J.; Calvino, J.J.; Lopez-Cartes, C.; Perez-Omil, J.A.; Rodriguez-Izquierdo, J.M. The interpretation of HREM images of supported metal catalysts using image simulation: Profile view images. Ultramicroscopy 1998, 72, 135-164. [CrossRef]

52. Gao, N.; Zhou, H.; Tan, H.; Qi, R.; Chen, J.; Zhang, S.; Xu, J. Turn-on fluorescence detection of cysteine with glutathione protected silver nanoclusters. Methods Appl. Fluoresc. 2019, 7, 034004. [CrossRef]

53. Enea, M.; Pereira, E.; Costa, J.; Soares, M.E.; da Silva, D.D.; Bastos, M.d.L.; Carmo, H.F. Cellular uptake and toxicity of gold nanoparticles on two distinct hepatic cell models. Toxicol. Vitr. 2021, 70, 105046. [CrossRef] [PubMed]

54. Maniglio, D.; Benetti, F.; Minati, L.; Jovicich, J.; Valentini, A.; Speranza, G.; Migliaresi, C. Theranostic gold-magnetite hybrid nanoparticles for MRI-guided radiosensitization. Nanotechnology 2018, 29, 315101. [CrossRef]

55. Guzman, F.V.; Mercadal, P.A.; Coronado, E.A.; Encina, E.R. Near-Field Enhancement Contribution to the Photoactivity in Magnetite-Gold Hybrid Nanostructures. J. Phys. Chem. C 2019, 123, 29891-29899. [CrossRef]

56. Bhatia, P.; Verma, S.S.; Sinha, M.M. Optical Properties Simulation of Magneto-Plasmonic Alloys Nanostructures. Plasmonics 2019, 14, 611-622. [CrossRef]

57. Parani, S.; Oluwafemi, O.S. Selective and sensitive fluorescent nanoprobe based on AgInS2-ZnS quantum dots for the rapid detection of Cr (III) ions in the midst of interfering ions. Nanotechnology 2020, 31, 395501. [CrossRef] [PubMed]

58. Santos, M.C.; Seabra, A.B.; Pelegrino, M.T.; Haddad, P.S. Synthesis, characterization and cytotoxicity of glutathione- and PEG-glutathione-superparamagnetic iron oxide nanoparticles for nitric oxide delivery. Appl. Surf. Sci. 2016, 367, 26-35. [CrossRef]

59. Dheyab, M.A.; Aziz, A.A.; Jameel, M.S.; Noqta, O.A.; Khaniabadi, P.M.; Mehrdel, B. Simple rapid stabilization method through citric acid modification for magnetite nanoparticles. Sci. Rep. 2020, 10, 10793. [CrossRef]

60. Gomes, S.A.O.; Vieira, C.S.; Almeida, D.B.; Santos-Mallet, J.R.; Menna-Barreto, R.F.S.; Cesar, C.L.; Feder, D. CdTe and CdSe Quantum Dots Cytotoxicity: A Comparative Study on Microorganisms. Sensors 2011, 11, 11664-11678. [CrossRef] 